\title{
Formalismo de Hamilton-Jacobi à la Carathéodory. Parte 2: sistemas singulares
}

(The Hamilton-Jacobi formalism à la Carathéodory. Part 2: singular systems)

\author{
M.C. Bertin ${ }^{1}$, B.M. Pimentel ${ }^{1}$ e P.J. Pompeia ${ }^{2}$ \\ ${ }^{1}$ Instituto de Física Teórica, Universidade Estadual Paulista "Júlio de Mesquita Filho", São Paulo, SP, Brasil \\ ${ }^{2}$ Theoretical Physics Institute, University of Alberta, Edmonton, Alberta, Canada \\ Recebido em 15/2/2008; Aceito em 11/3/2008; Publicado em 8/10/2008
}

Na segunda parte do artigo publicado na Revista Brasileira de Ensino de Física 29, 393 (2007) trataremos do formalismo de Hamilton-Jacobi para sistemas singulares. Mostraremos como é possível generalizar o procedimento de Carathéodory para Lagrangianas cuja matriz Hessiana é singular e construir um procedimento de análise de vínculos a partir desse formalismo.

Palavras-chave: equação de Hamilton-Jacobi, sistemas singulares, vínculos.

In the second part of the paper published on Revista Brasileira de Ensino de Física 29, 393 (2007) we will show how the Hamilton-Jacobi formalism works for singular systems. We will bring the generalization of the Carathéodory's method for Lagrangians whose Hessian matrix is singular. We will also build a procedure of constraint analysis of such systems based on the formalism.

Keywords: Hamilton-Jacobi equation, singular systems, constraints.

\section{Introdução}

Na primeira parte deste trabalho [1], mostramos como o formalismo de Hamilton-Jacobi (HJ) emerge de forma natural a partir do princípio de Hamilton com o método de Lagrangianas equivalentes de Carathéodory. O que fizemos foi tentar encontrar uma função $S(q, t)$ tal que $\bar{L}=L-d S / d t$ seja um extremo em uma trajetória dinâmica $C$ no espaço de configuração. Se essa função existe ela deve obedecer à equação ${ }^{2}$

$$
\frac{\partial S}{\partial t}+p_{i} \dot{q}^{i}-L=0, \quad\{i\}=\{1, \ldots, n\},
$$

em que $p_{i}=\partial S / \partial q^{i}$ e as velocidades $\dot{q}^{i}$ devem ser escritas em função das coordenadas e dos momentos, a partir da definição dos momentos conjugados

$$
p_{i} \equiv \frac{\partial L}{\partial \dot{q}^{i}}
$$

Devemos ser capazes de inverter essas relações para encontrar as velocidades, mas isso pode ser feito apenas se a condição Hessiana,

$$
\operatorname{det}\left(\frac{\partial^{2} L}{\partial \dot{q}^{i} \partial \dot{q}^{j}}\right) \neq 0,
$$

for satisfeita. O formalismo de HJ que desenvolvemos então exige que essa condição se cumpra. Por fim, a função $S$ deve obedecer à equação diferencial parcial (EDP) de primeira ordem

$$
\frac{\partial S}{\partial t}+H(t, q, \nabla S)=0
$$

ou seja, a equação de Hamilton-Jacobi. Os sistemas que obedecem à condição Hessiana são sistemas regulares e, como vimos, muitos sistemas mecânicos de interesse são desse tipo, como a partícula livre e o oscilador harmônico. Na mecânica clássica escrevemos a função Lagrangiana como a diferença entre a energia cinética e potencial, de modo que sempre que a energia cinética for quadrática nas velocidades em todas as coordenadas vemos claramente que a relação (2) será inversível.

Contudo, existem sistemas na natureza, tanto na mecânica quanto em teorias de campos, cujas Lagrangianas violam a condição (3). Esses são sistemas singulares. À primeira vista a violação da condição Hessiana

\footnotetext{
${ }^{1}$ E-mail: pimentel@ift.unesp.br.

${ }^{2}$ Neste trabalho empregaremos a convenção de soma, em que índices repetidos representam soma sobre os valores desses índices. Por exemplo, na expressão (1), $p_{i} \dot{q}^{i}$ deve ser compreendido por ${ }_{i=1}^{n} p_{i} \dot{q}^{i}$. 
impede que uma equação de HJ possa ser escrita a partir da relação (1), em razão da não existência de uma função Hamiltoniana, em (4), que seja única em todo o espaço de configuração. Nosso objetivo é, portanto, encontrar uma generalização do formalismo de HJ para lidar com o problema da violação da condição Hessiana.

$\mathrm{Na}$ primeira parte vimos também que o formalismo Hamiltoniano emerge naturalmente do formalismo de Hamilton-Jacobi: as equações canônicas de Hamilton são parte das equações características relacionadas à equação de HJ. Portanto, a questão da existência de um formalismo canônico para sistemas de Hessiana singular é intimamente ligada ao problema da existência de uma formulação de HJ. Dirac [2], na década de 1950, foi o primeiro a mostrar que o formalismo Hamiltoniano pode ser obtido para teorias singulares, com a introdução do método Hamiltoniano que levou seu nome. Com esse método uma questão ainda mais fundamental começou a ser discutida e ainda hoje é objeto de estudo: como quantizar esses sistemas? Desde que a quantização de um sistema envolve o conhecimento de sua estrutura canônica, o estudo de sistemas singulares torna-se fundamental. ${ }^{3}$

Nessa segunda parte traremos a versão de HamiltonJacobi desse problema, ou seja, como tratar classicamente os vínculos a partir desse formalismo. A princípio introduziremos a noção de Lagrangianas singulares e a razão pela qual sistemas com vínculos pressupõem redução de graus de liberdade. Em seguida traremos o desenvolvimento de Carathéodory, tal como mostrado na primeira parte, desta vez adaptado a sistemas singulares. Com isso encontraremos que sistemas vinculados obedecem não só a uma, mas a um conjunto de equações diferenciais parciais (EDP). Deduziremos as equações características desse sistema e nos preocuparemos em mostrar sua integrabilidade. Mostraremos como analisar as condições de integrabilidade e como encontrar equações de movimento a partir de três aplicações.

\section{Lagrangianas singulares}

Nosso primeiro passo será tentar compreender melhor o papel da condição Hessiana na resolução de um sistema dinâmico e as conseqüências de sua quebra. Vamos começar com as equações de Euler-Lagrange (EL)

$$
\frac{\partial L}{\partial q^{i}}-\frac{d}{d t}\left(\frac{\partial L}{\partial \dot{q}^{i}}\right)=0 .
$$

A princípio, vamos supor que a Lagrangiana seja independente do tempo, mas uma estensão imediata pode ser vista se $t$ estiver explicitamente presente. Vamos reescrever essas equações expandindo o termo da derivada temporal:

$$
\frac{\partial L}{\partial q^{i}}-\frac{\partial^{2} L}{\partial \dot{q}^{i} \partial q^{j}} \dot{q}^{j}-\frac{\partial^{2} L}{\partial \dot{q}^{i} \partial \dot{q}^{j}} \ddot{q}^{j}=0 .
$$

Podemos ver por essa forma que as equações de EL são equações diferenciais ordinárias (EDO) de segunda ordem nas coordenadas. Podemos, ainda, colocar essa equação em forma análoga às equações da segunda lei de Newton. De fato, as equações de EL são as equações de Newton escritas em função das coordenadas generalizadas. ${ }^{4}$ Isso pode ser observado se escrevermos

$$
\frac{\partial^{2} L}{\partial \dot{q}^{i} \partial \dot{q}^{j}} \ddot{q}^{j}=\frac{\partial L}{\partial q^{i}}-\frac{\partial^{2} L}{\partial \dot{q}^{i} \partial q^{j}} \dot{q}^{j} .
$$

Essa última equação sugere uma relação matricial,

$$
(W)_{i j} \ddot{q}^{j}=F_{i}(q, \dot{q}),
$$

na qual à esquerda figura a matriz Hessiana $W$. A condição Hessiana implica na existência da inversa da matriz $W$. Se ela for satisfeita, basta resolver o sistema de equações

$$
\ddot{q}^{i}=\left(W^{-1}\right)^{i j} F_{j} .
$$

No geral precisaremos de expressões para as velocidades $\dot{q}^{i}$ em $F_{i}$ para desacoplar as equações, mas podemos mostrar que se a condição Hessiana for satisfeita isso sempre é possível sem a imposição de condições extras. $^{5}$

Agora vamos considerar um sistema singular cuja Lagrangiana é da forma $L(x, y, \dot{x})$, que depende da coordenada $x$, da velocidade $\dot{x}$, mas apenas da coordenada $y$ [18]. A Hessiana desse sistema é singular. Coordenadas como $y$, que aparecem explicitamente na Lagrangiana sem que suas respectivas derivadas superiores apareçam, são chamadas coordenadas degeneradas. ${ }^{6}$ Para este exemplo temos as equações de EL

$$
\frac{\partial^{2} L}{\partial \dot{x}^{2}} \ddot{x}=\frac{\partial L}{\partial x}-\frac{\partial^{2} L}{\partial \dot{x} \partial x} \dot{x}-\frac{\partial^{2} L}{\partial \dot{x} \partial y} \dot{y}
$$

\footnotetext{
${ }^{3}$ Outros trabalhos relevantes no início do desenvolvimento da análise de sistemas singulares podem ser lidos em Anderson and Begmann [3], Dirac [4], Bergmann and Goldberg [5], Dirac [6] e DeWitt (não publicado. Veja a Ref. [11], p. 7.), trabalhos que ajudaram a esclarecer e a refinar diversos aspectos do método original. Na década seguinte um tratamento expandido de sistemas Hamiltonianos singulares foi estabelecido por Dirac, [7]. Outros aspectos foram tratados por diversos autores desde então, como Kundt [8] e Shanmugadhasan [9] e [10]. Boas referências para iniciar o estudo ao método Hamiltoniano são as Refs. [7], [11], [12] e [13].

${ }^{4}$ As Eqs. (5) pressupõem que as forças generalizadas possam ser escritas a partir do gradiente de um potencial. Assim, as equações dinâmicas de Newton, $F^{i}={ }_{i} m_{i} a^{i}$, são mais gerais que as equações de EL pois as primeiras admitem forças não conservativas. No entanto, há uma versão das equações de EL que admite forças generalizadas não conservativas. Neste trabalho, contudo, consideraremos apenas sistemas cujas forças sejam derivadas de potenciais. Veja Ref. [14], cap. 9, Ref. [15], caps. 1 e 2, Ref. [16], cap. 5 e Ref. [17], cap. 2 .

${ }^{5} \mathrm{O}$ leitor pode se convencer disso por um exemplo geral de uma Lagrangiana do tipo $L\left[q^{i},\left(\dot{q}^{i}\right)^{2}\right]$.

${ }^{6}$ Também chamadas ignoráveis.
} 


$$
\frac{\partial L}{\partial y}=0
$$

A primeira equação é uma equação dinâmica para $x$, mas para resolvê-la precisamos de duas condições. A primeira é que $\partial^{2} L / \partial \dot{x}^{2}$ seja não nulo. A segunda é uma expressão para $\dot{y}$ em termos de $x$ e $\dot{x}$. É possível notar que não existem equações dinâmicas para $y$ neste caso, ou seja, não há uma equação em que apareça uma derivada segunda de $y$. Temos apenas a segunda equação, que não fornece uma equação dinâmica e tampouco oferece a expressão para $\dot{y}$ que precisamos. Contudo, essa equação deve ser satisfeita pelo sistema. Ela é um vínculo Lagrangiano. O fato de o sistema ser vinculado pode ser também atestado pela matriz Hessiana

$$
W=\left(\frac{\partial^{2} L}{\partial \dot{q}^{i} \partial \dot{q}^{j}}\right)=\left(\begin{array}{cc}
\frac{\partial^{2} L}{\partial \dot{x}^{2}} & \frac{\partial^{2} L}{\partial \dot{x} \partial \dot{y}} \\
\frac{\partial^{2} L}{\partial \dot{y} \partial \dot{x}} & \frac{\partial^{2} L}{\partial \dot{y}^{2}}
\end{array}\right)=\left(\begin{array}{cc}
\frac{\partial^{2} L}{\partial \dot{x}^{2}} & 0 \\
0 & 0
\end{array}\right)
$$

Essa matriz é singular e não possui, portanto, uma inversa. De fato, ela possui um, ou talvez dois autovalores nulos dependendo do valor de $\partial^{2} L / \partial \dot{x}^{2}$. Caso este seja nulo, o sistema possuirá dois vínculos em vez de apenas um.

Sistemas singulares, cuja Hessiana não é inversível, têm a característica de serem sistemas com vínculos. Para resolver a Lagrangiana acima, por exemplo, precisamos de um meio para descobrir uma expressão para $\dot{y}$, ou reformular o sistema para que não precisemos dessa expressão. Outros exemplos, existentes na natureza, mostram-se ainda mais complexos: exigem formalismos capazes de lidar com a singularidade da matriz Hessiana. Adiante veremos como o formalismo de HJ abriga naturalmente esse tipo de sistema.

\section{O sistema de EDP de Hamilton- Jacobi}

O quadro completo de Carathéodory, aquele que nos diz que uma função $S(q, t)$ capaz de extremizar $\bar{L}$ representa uma família de superfícies ortogonal a uma congruência de curvas, solução de um sistema de equações diferenciais ordinárias, continua válido quando o sistema é singular. Vamos impor esse cenário como ponto de partida do formalismo. Assim, vamos partir das condições [1]

$$
\frac{\partial S}{\partial t}+p_{i} \dot{q}^{i}-L=0, \quad p_{i}=\frac{\partial S}{\partial q^{i}} .
$$

Queremos analisar sistemas que violam a condição Hessiana. Então, vamos supor que nossa Lagrangiana viole a condição Hessiana, ou seja,

$$
\operatorname{det} W=\operatorname{det}\left(\frac{\partial^{2} L}{\partial \dot{q}^{i} \partial \dot{q}^{j}}\right)=0 .
$$

A matriz Hessiana é simétrica e, portanto, sempre diagonalizável. Vamos supor que ela possua um número $m$ de autovalores não nulos e um número $k$ de autovalores nulos, tal que $m+k=n$, a dimensão do espaço de configuração. Dizemos que uma matriz nessas condições tem posto $m$. Existirá, neste caso, uma submatriz $m \times m$ contida na Hessiana que terá determinante não nulo e, por conseqüência, será inversível. Com essa situação temos uma divisão entre as coordenadas. O primeiro conjunto é aquele referente à parte inversível da Hessiana. Para estes é possível encontrar uma expressão para as velocidades em termos das posições e dos momentos

$$
\begin{gathered}
p_{a}=\frac{\partial L}{\partial \dot{q}^{a}} \longrightarrow \dot{q}^{a}=\eta^{a}\left(q^{i}, p_{b}\right), \\
\{a, b\}=\{1, \ldots, m\} .
\end{gathered}
$$

O segundo conjunto de coordenadas é aquele que se refere ao setor nulo da matriz. Para estes não é possível encontrar expressões para as respectivas velocidades pois as equações

$$
p_{z}=\frac{\partial L}{\partial \dot{q}^{z}}, \quad\{x, y, z\}=\{1, \ldots, k\},
$$

não podem ser invertidas. As Eq. (13) serão consideradas vínculos da teoria. Escreveremos esses vínculos da seguinte forma

$$
\phi_{z} \equiv p_{z}+H_{z}\left(q^{i}, \eta^{a}\right)=0 \quad \longrightarrow \quad H_{z} \equiv-\frac{\partial L}{\partial \dot{q}^{z}} .
$$

Assumiremos que a Lagrangiana é bem comportada, no sentido que as funções $H_{z}$ não dependam de $\dot{q}^{z}$, embora essa dependência possa existir sem que seja possível uma Hessiana inversível. Com essa divisão entre as coordenadas $\left\{q^{a}\right\}$ e $\left\{q^{z}\right\}$ podemos analisar a Eq. (10), que toma a forma

$$
\frac{\partial S}{\partial t}+p_{a} \eta^{a}\left(q^{i}, p_{b}\right)+p_{z} \dot{q}^{z}-L=0 .
$$

O problema das velocidades $\dot{q}^{z}$ ainda persiste, mas apenas aparentemente. Vamos analisar a derivada

$$
\frac{\partial}{\partial \dot{q}^{y}}\left(p_{a} \eta^{a}\left(q^{i}, p_{b}\right)+p_{z} \dot{q}^{z}-L\right)=p_{y}-\frac{\partial L}{\partial \dot{q}^{y}}=\phi_{y}=0 .
$$

Ou seja, se os vínculos forem respeitados, os termos na Eq. (15), que dariam origem à função Hamiltoniana Canônica

$$
H_{0} \equiv p_{a} \eta^{a}\left(q^{i}, p_{b}\right)+p_{z} \dot{q}^{z}-L,
$$

não dependerão das velocidades não inversíveis. Isso quer dizer que na região em que os vínculos são satisfeitos essa Hamiltoniana é bem definida. A Eq. (10) pode ser escrita, então, por

$$
\phi_{0} \equiv \frac{\partial S}{\partial t}+H_{0}\left(q^{i}, \eta^{a}\right)=0
$$


Ora, podemos definir como $p_{0}$ a derivada de $S$ com relação a $t$ e substituir as expressões para as funções $\eta$. Assim, temos as equações

$$
\begin{aligned}
& \phi_{0} \equiv p_{0}+H_{0}\left(q^{i}, p_{a}\right)=0, \\
& \phi_{z} \equiv p_{z}+H_{z}\left(q^{i}, p_{a}\right)=0 .
\end{aligned}
$$

Podemos escrever de forma unificada

$$
\phi_{\alpha} \equiv p_{\alpha}+H_{\alpha}\left(q^{i}, p_{a}\right)=0, \quad\{\alpha, \beta\}=\{0,1, \ldots, k\} .
$$

Como ainda devem valer as condições $p_{i}=\partial S / \partial q^{i}$, essas equações formam um conjunto de EDP. Esse sistema de equações é chamado de sistema de EDP de Hamilton-Jacobi. ${ }^{7}$

\section{Equações características}

Novamente somos capazes, a partir do procedimento de Cauchy [19, 20], de encontrar equações diferenciais totais (EDT), cuja solução nos fornece uma congruência de curvas no espaço de configuração, a partir das EDP de Hamilton-Jacobi. Este sistema pode ser escrito na forma

$$
\begin{gathered}
\phi_{\alpha}\left(q^{a}, q^{\alpha}, p_{a}, p_{\alpha}\right)=0 \longrightarrow\{\alpha\}=\{0, \ldots, k\}, \\
q^{0} \equiv t, \quad p_{0} \equiv \frac{\partial S}{\partial q^{0}} .
\end{gathered}
$$

A princípio vamos supor que este sistema seja o mais geral possível. Em conjunto com este sistema está implícito que as condições

$$
p_{\alpha}=\frac{\partial}{\partial q^{\alpha}} S\left(q^{a}, q^{\alpha}\right)
$$

são satisfeitas. Vamos tomar as diferenciais

$$
d p_{a}=\frac{\partial^{2} S}{\partial q^{a} \partial q^{b}} d q^{b}+\frac{\partial^{2} S}{\partial q^{a} \partial q^{\alpha}} d q^{\alpha} .
$$

Devemos guardar essas equações e tomar a derivada da Eq. (19) com relação a $q^{a}$

$$
\frac{\partial \phi_{\alpha}}{\partial q^{a}}+\frac{\partial \phi_{\alpha}}{\partial p_{\beta}} \frac{\partial p_{\beta}}{\partial q^{a}}+\frac{\partial \phi_{\alpha}}{\partial p_{b}} \frac{\partial p_{b}}{\partial q^{a}}=0 .
$$

Agora vamos particularizar o sistema. Estamos interessados no caso em que $\phi_{\alpha}$ sejam funções lineares dos momentos $p_{\alpha}$, assim como mostra a Eq. (18). Assim, $\partial \phi_{\alpha} / \partial p_{\beta}=\delta_{\alpha}^{\beta}$. Com essa constatação podemos escrever as equações

$$
\frac{\partial p_{\alpha}}{\partial q^{a}}=\frac{\partial^{2} S}{\partial q^{\alpha} \partial q^{a}}=-\frac{\partial \phi_{\alpha}}{\partial q^{a}}-\frac{\partial \phi_{\alpha}}{\partial p_{b}} \frac{\partial p_{b}}{\partial q^{a}} .
$$

Substituindo essas equações na Eq. (21)

$$
d p_{a}=\frac{\partial^{2} S}{\partial q^{a} \partial q^{b}} d q^{b}-\left[\frac{\partial \phi_{\alpha}}{\partial q^{a}}+\frac{\partial \phi_{\alpha}}{\partial p_{b}} \frac{\partial p_{b}}{\partial q^{a}}\right] d q^{\alpha} .
$$

Também devemos preservar essas últimas equações. A partir da definição da Hamiltoniana canônica temos

$$
\phi_{0}=p_{0}+H_{0}=p_{0}+p_{a} \dot{q}^{a}+p_{z} \dot{q}^{z}-L .
$$

Podemos usar os vínculos $p_{z}=-H_{z}$ e derivar essa expressão com relação aos momentos $p_{b}$

$$
\frac{\partial \phi_{0}}{\partial p_{b}}=\dot{q}^{b}-\frac{\partial H_{z}}{\partial p_{b}} \dot{q}^{z}=\dot{q}^{b}-\frac{\partial \phi_{z}}{\partial p_{b}} \dot{q}^{z} .
$$

Vamos reordenar essa equação introduzindo o termo $\dot{q}^{0}$, que nada mais é que 1 . Assim

$$
\dot{q}^{b}=\frac{\partial \phi_{0}}{\partial p_{b}} \dot{q}^{0}+\frac{\partial \phi_{z}}{\partial p_{b}} \dot{q}^{z} \longrightarrow d q^{b}=\frac{\partial \phi_{\alpha}}{\partial p_{b}} d q^{\alpha}
$$

Na Eq. (24), vamos substituir a Eq. (25)

$$
d p_{a}=\frac{\partial p_{a}}{\partial q^{b}} \frac{\partial \phi_{\alpha}}{\partial p_{b}} d q^{\alpha}-\left[\frac{\partial \phi_{\alpha}}{\partial q^{a}}+\frac{\partial \phi_{\alpha}}{\partial p_{b}} \frac{\partial p_{b}}{\partial q^{a}}\right] d q^{\alpha}
$$

Como $p_{b}=\partial S / \partial q^{b}$, temos que $\partial p_{b} / \partial q^{a}=\partial p_{a} / \partial q^{b}$. Com isso

$$
d p_{a}=-\frac{\partial \phi_{\alpha}}{\partial q^{a}} d q^{\alpha}
$$

Também podemos tomar a diferencial da função $S\left(q^{a}, q^{\alpha}\right)$

$$
d S=\frac{\partial S}{\partial q^{a}} d q^{a}+\frac{\partial S}{\partial q^{\alpha}} d q^{\alpha} .
$$

Usando a Eq. (25)

$d S=\frac{\partial S}{\partial q^{a}} \frac{\partial \phi_{\alpha}}{\partial p_{a}} d q^{\alpha}+\frac{\partial S}{\partial q^{\alpha}} d q^{\alpha}=\left[p_{a} \frac{\partial H_{\alpha}}{\partial p_{a}}-H_{\alpha}\right] d q^{\alpha}$,

que em forma integral nos fornece a equação

$$
S=\int\left[p_{a} d q^{a}-H_{\alpha} d q^{\alpha}\right],
$$

em que empregamos a Eq. (25) novamente.

Por fim, temos por equações características do sistema

\footnotetext{
${ }^{7}$ De fato, podemos formular a dinâmica de modo que um sistema regular seja um caso particular de um sistema singular. Para isso basta uma reparametrização arbitrária e a introdução do tempo como variável dinâmica. Nesse caso um sistema regular torna-se singular e seu vínculo vem a ser a equação de HJ, $\partial S / \partial t+H_{0}=0$. Esse tipo de sistema é sempre singular pois o tempo torna-se uma coordenada degenerada. É natural, sob este ponto de vista, escrever de forma unificada os vínculos da Eqs. (14) e (17).
} 


$$
\begin{aligned}
& d q^{a}=\frac{\partial \phi_{\alpha}}{\partial p_{a}} d q^{\alpha} \\
& d p_{a}=-\frac{\partial \phi_{\alpha}}{\partial q^{a}} d q^{\alpha} \\
& d S=\left[p_{a} \frac{\partial H_{\alpha}}{\partial p_{a}}-H_{\alpha}\right] d q^{\alpha} .
\end{aligned}
$$

Essas equações definem um espaço de fase de dimensão $2 m$ cujas coordenadas são as variáveis $\left(q^{a}, p_{a}\right)$. Como solução dessas equações temos uma congruência de curvas nesse espaço e as curvas são parametrizadas pelas variáveis $q^{\alpha}$. Isso indica que, no espaço de configuração, as trajetórias dinâmicas restringem-se ao espaço das variáveis $q^{a}$. A menos de condições iniciais a solução do sistema no espaço de configuração consiste em trajetórias cujas equações paramétricas são do tipo $q^{a}=q^{a}\left(q^{z}, t\right)$ e, portanto, as variáveis $q^{z}$ são parâmetros em condições de igualdade a $t$.

Observáveis físicos devem, por consistência, ser funções desse espaço de fase reduzido. A evolução de um observável agora não é ditada apenas por um parâmetro de evolução, mas por um conjunto deles. Chamaremos o conjunto de parâmetros $q^{\alpha}$ de variáveis independentes do sistema. A evolução de um observável $F\left(q^{a}, p_{a}, q^{\alpha}\right)$ é dada por

$$
d F=\frac{\partial F}{\partial q^{\alpha}} d q^{\alpha}+\frac{\partial F}{\partial q^{a}} d q^{a}+\frac{\partial F}{\partial p_{a}} d p_{a} .
$$

Com o auxílio das equações características,

$$
d F=\frac{\partial F}{\partial q^{\alpha}} d q^{\alpha}+\left[\frac{\partial F}{\partial q^{a}} \frac{\partial \phi_{\alpha}}{\partial p_{a}}-\frac{\partial F}{\partial p_{a}} \frac{\partial \phi_{\alpha}}{\partial q^{a}}\right] d q^{\alpha} .
$$

Note que podemos definir um Parênteses de Poisson (PP) em termos de todas as variáveis do problema

$$
\begin{aligned}
& \{F, G\} \equiv \frac{\partial F}{\partial q^{a}} \frac{\partial G}{\partial p_{a}}-\frac{\partial F}{\partial p_{a}} \frac{\partial G}{\partial q^{a}}+\frac{\partial F}{\partial q^{\alpha}} \frac{\partial G}{\partial p_{\alpha}}- \\
& \frac{\partial F}{\partial p_{\alpha}} \frac{\partial G}{\partial q^{\alpha}}=\frac{\partial F}{\partial q^{\alpha^{\prime}}} \frac{\partial G}{\partial p_{\alpha^{\prime}}}-\frac{\partial F}{\partial p_{\alpha^{\prime}}} \frac{\partial G}{\partial q^{\alpha^{\prime}}} \\
& \left\{\alpha^{\prime}\right\}=\{0,1, \ldots, n\}
\end{aligned}
$$

com o qual podemos escrever a diferencial

$$
d F=\left\{F, \phi_{\alpha}\right\} d q^{\alpha} .
$$

Este parênteses obedece às mesmas propriedades de um PP para um sistema regular, particularmente a antissimetria, a bilinearidade e a identidade de Jacobi. A forma da Eq. (34) é simples por conter em sua estrutura de PP os parâmetros da teoria. Contudo, só podemos definir verdadeiramente um espaço de fase para o conjunto de varíáveis $\left(q^{a}, p_{a}\right){ }^{8}$ Essas são chamadas variáveis dependentes, pois serão funções das variáveis independentes. O espaço de fase construído com as variáveis dependentes é chamado espaço de fase reduzido.

Este quadro já poderia ser elucidado por uma análise da Eq. (28), que é uma ação canônica referente a um sistema com várias variáveis independentes. Um caso particular dessa ação é o caso em que apenas o tempo é tido como parâmetro, ou seja

$$
S=\int\left(p_{i} d q^{i}-H_{0} d t\right)
$$

Esta é a ação canônica da mecânica clássica. Já é bem conhecido o fato de que, igualada a zero a variação dessa ação com relação às variáveis $\left(q^{i}, p_{i}\right)$, encontramos as equações de Hamilton. Se aplicarmos o princípio de ação estacionária na Eq. (28), fazendo a variação com relação às variáveis $\left(q^{a}, p_{a}\right)$, encontraremos as equações características (29) e (30). Assim, a princípio, sistemas singulares são sistemas com várias variáveis independentes e, como já dissemos, sistemas regulares são apenas um caso particular de sistemas vinculados.

Dissemos "a princípio" porque há um detalhe que negligenciamos até agora. Assumimos o quadro de que existe uma família de superfícies sempre ortogonal a uma congruência de curvas no espaço de configuração, ou seja, dadas as Eqs. (18), não nos perguntamos se sempre existem soluções completas e, caso a resposta seja negativa, quais as condições necessárias para que exista pelo menos uma solução desse tipo.

\section{O quadro completo e condições de in- tegrabilidade}

Precisamos analisar melhor o quadro geométrico desse formalismo e o faremos com um sistema simples em $\mathbb{R}^{3}$. Vamos supor que exista uma congruência de curvas $C$ em uma região de $\mathbb{R}^{3}$ que seja ortogonal a uma família de superfícies bidimensionais $S(\mathbf{x})=\sigma$. Em particular, vamos começar analisando o que ocorre na vizinhança de um ponto $\mathbf{x}_{0}$, em que passam uma curva $C_{0}$ e uma superfície $S=\sigma_{0}$ (Fig. 1).

Em $\mathbf{x}_{0}$ podemos definir um vetor $\mathbf{v}_{0}$, tangente à curva $C_{0}$. Esse vetor é membro de um campo vetorial $\mathbf{v}=\mathbf{v}(\mathbf{x})$ que é tangente à congruência em todo ponto da região. No mesmo ponto passa a superfície

\footnotetext{
${ }^{8}$ Esta questão tem origem no fato de um espaço de fase dever possuir uma operação inversa ao Parênteses de Poisson que seja única. No caso do parênteses da Eq. (33) essa inversa não existe ou, na melhor das hipóteses, não será única. É possível mostrar, contudo, que sendo possível a separação entre as variáveis $q^{a}$ e $q^{\alpha}$, o parênteses definido por
}

$$
\{F, G\} \equiv \frac{\partial F}{\partial q^{a}} \frac{\partial G}{\partial p_{a}}-\frac{\partial F}{\partial p_{a}} \frac{\partial G}{\partial q^{a}}
$$

possui uma inversa e esta é única. Contudo, este tópico foge ao escopo deste trabalho. 
$\sigma_{0}$. Sendo de duas dimensões, podemos definir em $\mathbf{x}_{0}$ outros dois vetores linearmente independentes $\chi_{\alpha}$, em que $\{\alpha\}=\{1,2\}$, tangentes à superfície.

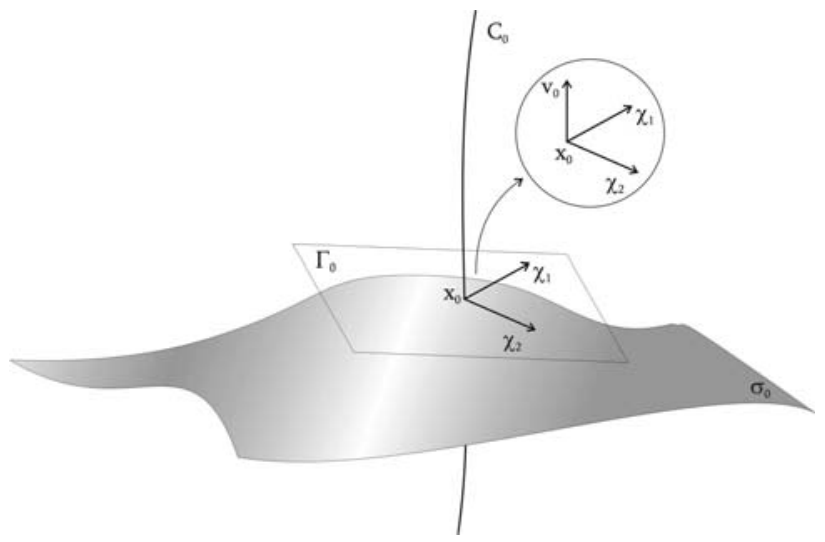

Figura 1 - A trajetória $C_{0}$ ortogonal à superfície $\sigma_{0}$.

Os vetores $\chi_{\alpha}$ podem ser usados como base para um espaço vetorial tangente à superfície $\sigma_{0}$ e, como estamos tratando de um caso em $\mathbb{R}^{3}$, eles definem um plano $\Gamma_{0}$ tangente à superfície em $\mathbf{x}_{0}$. Se, partindo do ponto $\mathbf{x}_{0}$, caminharmos para um outro ponto $\mathbf{x}_{1}$, encontraremos uma outra superfície, por exemplo $S=\sigma_{1}$, bem como uma outra curva $C_{1}$ e, sobre este ponto, encontramos um vetor $\mathbf{v}_{1}$ tangente a $C_{1}$ e, também, podemos construir analogamente um plano tangente a $\sigma_{1}, \Gamma_{1}$.

Podemos mostrar que qualquer base escolhida para $\Gamma_{1}$ é combinação linear dos vetores de base de $\Gamma_{0}$ e a razão é que $C_{0}$ e $C_{1}$ fazem parte da mesma congruência, bem como $\sigma_{0}$ e $\sigma_{1}$ fazem parte da mesma família. ${ }^{9}$ Assim, em todos os pontos da região, cada superfície da família $\sigma$ possui um plano tangente e a base vetorial para cada plano é univocamente determinada se conhecemos a base de um deles. Isso equivale a dizer que $\chi_{\alpha}$ também são membros de campos vetoriais $\chi_{\alpha}(\mathbf{x})$.

Dizemos que esse conjunto de planos forma uma distribuição, ${ }^{10}$ que chamaremos $\Gamma$. Trataremos essa distribuição como o espaço tangente à família de superfícies. Assim, os campos vetoriais $\chi_{\alpha}(\mathbf{x})$ serão tratados como base para toda a distribuição. Para que $C_{0}$ e $\sigma_{0}$ sejam transversos em $\mathbf{x}_{0}$ o produto escalar entre o vetor tangente à curva e os vetores tangentes à superfície deve ser nulo. Em uma afirmação um pouco mais geral, as equações

$$
\mathbf{v}(\mathbf{x}) \cdot \chi_{\alpha}(\mathbf{x})=0
$$

são as condições para que a congruência de curvas $C$ seja ortogonal à distribuição $\Gamma$.

No formalismo de HJ vimos que as componentes do momento conjugado são proporcionais ao gradiente da função $S$ que define a família de superfícies. Para este exemplo a afirmação equivalente é estabelecer

$$
\mathbf{v}(\mathbf{x})=\nabla S
$$

e, neste caso, as Eqs. (36) resumem-se a

$$
\chi_{\alpha}^{i} \partial_{i} S=0, \quad\{i\}=\{1,2,3\} .
$$

Essas equações garantem a transversalidade entre a congruência $C$ e a família de superfícies $S=\sigma$.

No caso de sistemas dinâmicos o quadro é análogo. Todo sistema de EDP de primeira ordem, como é o caso das equações de HJ, pode ser escrito na forma

$$
\begin{gathered}
X_{\alpha} S=\frac{\partial S}{\partial x^{\alpha}}+\chi_{\alpha}^{a} \frac{\partial S}{\partial y^{a}}=0, \quad\{a\}=\{1, \ldots, m\}, \\
\{\alpha\}=\{1, \ldots, k\},
\end{gathered}
$$

em que $\chi_{\alpha}^{a}$ são as $m$ componentes de $k$ vetores linearmente independentes $X_{\alpha}$. Podemos verificar que as características desse sistema formam as equações

$$
d y^{a}=\chi_{\alpha}^{a} d x^{\alpha}
$$

Neste caso as soluções possuem a forma $y^{a}=y^{a}\left(x^{\alpha}\right)$, sendo $x$ as variáveis independentes do sistema. Uma função $S(y, x)$, solução das Eqs. (39), representa uma família $S(y, x)=\sigma$ de superfícies em uma região do espaço cujas coordenadas são as variáveis dependentes $y$. Essa família é, em todo ponto onde ela possa ser definida, transversal a uma congruência de curvas $y^{a}=y^{a}\left(x^{\alpha}\right)$, solução das Eqs. (40). Os vínculos, na forma do sistema de EDP de HJ, são as equações que garantem a transversalidade entre a congruência de trajetórias e a família de superfícies. Os vetores $X_{\alpha}$, cujas componentes são $\chi_{\alpha}^{a}$, formam a base para a distribuição $\Gamma$ neste caso.

Para que este quadro se cumpra os vetores $X_{\alpha}$ devem formar uma base vetorial completa e linearmente independente (LI). Essa condição garante que os parâmetros $x^{\alpha}$ sejam independentes entre si. Caso nosso problema singular seja caracterizado por EDP que não formem uma base completa, a solução dessas equações também não será completa, no sentido que não dependerá de tantos parâmetros quantos forem as variáveis dependentes do sistema. Isso também afeta as equações características, no sentido que estas não serão completamente integráveis.

Para a análise de integrabilidade é conveniente tratar os vetores tangentes ao espaço como operadores diferenciais. Esses operadores agem sobre funções e resultam em outras funções do espaço de origem. Podemos escrever os vetores de base da distribuição como os operadores

$$
X_{\alpha}=\frac{\partial}{\partial x^{\alpha}}+\chi_{\alpha}^{a} \frac{\partial}{\partial y^{a}} .
$$

\footnotetext{
${ }^{9} \mathrm{O}$ fato de ambas as curvas serem da mesma congruência garante que dois vetores, pertencentes a cada curva, sejam membros de um mesmo campo vetorial. O mesmo ocorre com vetores tangentes a duas superfícies de uma mesma família.

${ }^{10}$ Não confundir com os objetos da teoria das distribuições.
} 
Agindo sobre funções do espaço de configuração esses vetores reultam em outras funções. Particularmente,

$$
\phi_{\alpha} \equiv X_{\alpha}(S)=0
$$

são as equações de HJ.

A estrutura de colchetes definida por

$$
\left[X_{\alpha}, X_{\beta}\right](F) \equiv X_{\alpha}\left\{X_{\beta}(F)\right\}-X_{\beta}\left\{X_{\alpha}(F)\right\}
$$

é chamada colchetes de Lie. ${ }^{11} \mathrm{Na}$ distribuição $\Gamma$ os colchetes de Lie tomados com quaisquer dois de seus membros deve ser também um membro da distribuição. Essa condição é chamada condição de integrabilidade de Frobenius. Se $X_{\alpha}$ constitui um conjunto completo de vetores LI, temos automaticamente satisfeita a equação

$$
\left[X_{\alpha}, X_{\beta}\right](F)=C_{\alpha \beta}^{\gamma} X_{\gamma}(F),
$$

ou seja, os vetores de base formam uma álgebra de Lie em que $C_{\alpha \beta}^{\gamma}$ são as constantes de estrutura.

Especificamente no caso de sistemas Lagrangianos singulares, o sistema de EDP são as equações de Hamilton-Jacobi

$$
\phi_{\alpha}=p_{\alpha}+H_{\alpha}\left(q^{a}, q^{\alpha}, p_{a}\right)=0, \quad p_{i}=\frac{\partial S}{\partial q^{i}},
$$

relacionadas às características

$$
d q^{a}=\left\{q^{a}, \phi_{\alpha}\right\} d q^{\alpha} .
$$

Portanto, as componentes dos vetores de base da distribuição pertinentes a esse sistema vêm a ser

$$
\chi_{\alpha}^{a}=\left\{q^{a}, \phi_{\alpha}\right\} .
$$

Com essas componentes temos, sendo $F$ uma função do espaço de configuração

$$
\begin{aligned}
X_{\alpha}(F) & =\chi_{\alpha}^{a} \frac{\partial F}{\partial q^{a}}=\left\{q^{a}, \phi_{\alpha}\right\} \frac{\partial F}{\partial q^{a}} \\
& =\delta_{b}^{a} \frac{\partial \phi_{\alpha}}{\partial p_{b}} \frac{\partial F}{\partial q^{a}}=\left\{F, \phi_{\alpha}\right\} .
\end{aligned}
$$

Por outro lado

$$
X_{\alpha}\left[X_{\beta}(F)\right]=X_{\alpha}\left(\left\{F, \phi_{\beta}\right\}\right)=\left\{\left\{F, \phi_{\beta}\right\}, \phi_{\alpha}\right\} .
$$

Isso nos permite escrever o seguinte resultado para os colchetes de Lie (usando a identidade de Jacobi para os PP)

$$
\begin{aligned}
{\left[X_{\alpha}, X_{\beta}\right](F)=} & \left\{\left\{F, \phi_{\beta}\right\}, \phi_{\alpha}\right\}-\left\{\left\{F, \phi_{\alpha}\right\}, \phi_{\beta}\right\} \\
= & -\left\{\left\{\phi_{\alpha}, F\right\}, \phi_{\beta}\right\}-\left\{\left\{\phi_{\beta}, \phi_{\alpha}\right\}, F\right\}- \\
& \left\{\left\{F, \phi_{\alpha}\right\}, \phi_{\beta}\right\} \\
= & \left\{\left\{\phi_{\alpha}, \phi_{\beta}\right\}, F\right\} .
\end{aligned}
$$

Com isso temos, a partir das condições de integrabilidade,

$$
\left\{\phi_{\alpha}, \phi_{\beta}\right\}=C_{\alpha \beta}^{\gamma} \phi_{\gamma}
$$

Portanto, os vínculos também devem obedecer a uma álgebra de Lie, desta vez com os parênteses de Poisson. Na mecânica clássica dizemos que os vínculos formam um sistema em involução. Todo sistema dinâmico é integrável se formos capazes de encontrar um conjunto de funções dinâmicas invariantes ao movimento que formem um conjunto involutivo.

Se um dado sistema de EDP não obedece identicamente as condições (51) podemos escolher forçar a integrabilidade do sistema. Essa ação equivale a assumir a existência de um subespaço em que a integrabilidade seja cumprida ou mesmo assumir que as variáveis independentes não sejam realmente independentes entre si. As próprias condições de integrabilidade, como equações impostas sobre o sistema original, podem revelar novos vínculos que completem o sistema de equações e/ou revelar a dependência entre as variáveis independentes. Nesse caso é melhor reformular as condições de integrabilidade de modo que

$$
d \phi_{\alpha}=\left\{\phi_{\alpha}, \phi_{\beta}\right\} d q^{\beta}=C_{\alpha \beta}^{\gamma} \phi_{\gamma} d q^{\beta}=0,
$$

em que assumimos a validade das equações $\phi_{\alpha}=0$. Portanto, as equações $d \phi_{\alpha}=0$ também devem ser tomadas como condições sobre o sistema. Essas condições exigem que os vínculos sejam mantidos inalterados durante a trajetória dinâmica e garantem a existência de soluções completas para o sistema de HJ, bem como a integrabilidade das equações características. Formuladas com essa interpretação, a condição de integrabilidade de Frobenius nos garante um meio de encontrar um conjunto involutivo de integrais de movimento.

O procedimento a seguir agora depende fortemente do sistema em estudo, especificamente da existência ou não de um conjunto ou subconjunto de vínculos em involução. O melhor agora é analisarmos alguns exemplos. Vamos começar com a aplicação da partícula livre em uma esfera, que nos fornecerá um exemplo em que o conjunto de vínculos não é involutivo.

\footnotetext{
${ }^{11}$ Os colchetes de Lie são operadores bilineares que agem em vetores e levam a outros vetores. Eles dizem como um campo vetorial varia com relação a uma curva gerada por outro campo vetorial. Por exemplo, considere dois vetores $X=\alpha^{i}(\mathbf{x}) \partial_{i}$ e $Y=\beta^{i}(\mathbf{x}) \partial_{i}$, em que $\alpha^{i}$ e $\beta^{i}$ são as componentes dos campos dado um sistema de coordenadas retangular $x^{i}$. Tanto $X$ quanto $Y$ podem ser usados para gerar curvas dinâmicas no espaço. Essas curvas seriam soluções das equações $\dot{x}^{i}=\alpha^{i}(\mathbf{x})$ e $\dot{x}^{i}=\beta^{i}(\mathbf{x})$. Se quisermos saber como varia o campo $X$ sobre a curva gerada por $Y$ basta resolver $[X, Y]$. Por ser antissimétrico, o resultado nos dá a negativa da variação de $Y$ com relação à curva de $X$. Este é um tópico de geometria diferencial referente à derivada de Lie, que pode ser estudado, não sem antes passar por tópicos introdutórios, Refs. [21, 22] e também na Ref. [17], cap. 3.
} 


\section{Partícula livre em uma esfera}

Na mecânica clássica o exemplo mais geral de um sistema vinculado é a partícula livre restrita a uma superfície. De fato, sistemas regulares bem conhecidos também podem ser descritos dessa forma, como por exemplo o pêndulo simples, que consiste em uma partícula livre no potencial gravitacional cujo comprimento da haste é constante. É possível um tratamento bastante geral deste problema, mas visando a clareza do formalismo vamos nos concentrar em resolvê-lo no espaço $\mathbb{R}^{3}$, sendo a superfície uma esfera.

Consideraremos um sistema no espaço euclidiano $\mathbb{R}^{3}$, que consiste de uma partícula livre restrita à superfície de uma esfera. Essa superfície é definida, no sistema cartesiano, pela equação $\psi \equiv x^{2}+y^{2}+z^{2}-r^{2}=0$. Em nossa notação, $\mathbf{x}$ representa um ponto em $\mathbb{R}^{3}$ cujas coordenadas são o conjunto de números coordenados $(x, y, z)$. Como estamos tratando de um espaço euclidiano, x pode também representar um vetor posição. A Lagrangiana da partícula livre consiste apenas da energia cinética, mas devemos implementar o vínculo $\psi=0$ usando multiplicadores de Lagrange, de modo que a Lagrangiana do sistema se escreve por

$$
L(\mathbf{x}, \mathbf{v})=\frac{1}{2} m \mathbf{v}^{2}+u \psi(\mathbf{x})
$$

na qual $\mathbf{v} \equiv \dot{\mathbf{x}}$ representa o vetor velocidade da partícula.

Vamos escrever o vetor momento conjugado a partir dessa função

$$
\mathbf{p}=\frac{\partial L}{\partial \mathbf{v}}=m \mathbf{v}
$$

E com estes, as equações de Euler-Lagrangre desse sistema, $\dot{\mathbf{p}}-\nabla L=0$

$$
m \mathbf{a}-u \nabla \psi=0,
$$

em que $\nabla$ representa o operador gradiente e a o vetor aceleração $(\mathbf{a}=\dot{\mathbf{v}})$. A expressão $u \nabla \psi$ é reconhecida como a força que mantém o sistema sobre a superfície. No entanto, como o parâmetro $u$ se mantém indeterminado, essa força é parametrizada, ou seja, várias configurações são permitidas. ${ }^{12}$

Para utilizar o formalismo de HJ construiremos um espaço de configuração estendido em que as variáveis dinâmicas serão as posições x e também o parâmetro $u$. Vimos que existem 3 momentos conjugados às posições, dados pelas expressões inversíveis $\mathbf{p}=m \mathbf{v}$. O momento referente à variável $u, p_{u}$, é nulo, pois a Lagrangiana não depende da velocidade relativa a essa variável. Assim, da definição dos momentos encontramos 3 velocidades e 1 vínculo

$$
\begin{gathered}
\mathbf{v}=\mathbf{p} / m, \\
\phi_{1} \equiv p_{u}=0 .
\end{gathered}
$$

Com essas equações podemos construir a Hamiltoniana canônica do sistema

$$
H_{0}=\mathbf{p} \cdot \mathbf{v}+\pi \dot{u}-L=\frac{\mathbf{p}^{2}}{2 m}-u \psi .
$$

Portanto, além do vínculo (57), o sistema obedece a uma equação de HJ que envolve a Hamiltoniana canônica (58), de modo que o sistema consiste nas equações diferenciais parciais

$$
\begin{aligned}
& \phi_{0} \equiv p_{0}+H_{0}=0, \\
& \phi_{1} \equiv p_{u}=0 .
\end{aligned}
$$

Esse sistema tem como variáveis dependentes as posições $\mathbf{x}$ e como variáveis independentes os parâmetros $\left(q^{\alpha}\right)=(u, t)$. A evolução de uma função dinâmica qualquer $F\left(\mathbf{x}, \mathbf{p}, u, p_{u}\right)$ é dada, portanto, pela diferencial

$$
d F=\left\{F, \phi_{0}\right\} d t+\left\{F, \phi_{1}\right\} d u .
$$

Para o cálculo dos PP, usaremos os parênteses fundamentais

$$
\begin{array}{ll}
\{\mathbf{x}, \mathbf{p}\}=\mathbf{I}, \quad\{\mathbf{x}, \mathbf{x}\}=\{\mathbf{p}, \mathbf{p}\}=0, \\
\left\{u, p_{u}\right\}=1, \quad\{u, u\}=\left\{p_{u}, p_{u}\right\}=0,
\end{array}
$$

em que $\mathbf{I}$ é a matriz identidade $3 \times 3$.

O sistema de Eqs. (59) e (60) não é integrável, pois os vínculos não estão em involução. Isso pode ser visto pelo cálculo do PP

$$
\left\{\phi_{1}, \phi_{0}\right\}=\left\{p_{u},-u \psi\right\}=\psi
$$

que não é identicamente nulo, a princípio. Neste caso usaremos a diferencial (61) para verificar as condições de integrabilidade. Com relação à equação $\phi_{1}=0$,

$$
d \phi_{1}=\left\{\phi_{1}, \phi_{0}\right\} d t+\left\{\phi_{1}, \phi_{1}\right\} d u=\psi d t=0 .
$$

Portanto, para que o sistema seja integrável há que se obedecer mais uma equação,

$$
\psi=0 \text {. }
$$

Esta equação é aquela que define a esfera. A existência dessa condição indica que, ao sistema de equações (59) e (60), deve ser acrescida a equação

$$
\phi_{2} \equiv \psi=0 \text {. }
$$

\footnotetext{
${ }^{12} E$ É desejável este fato, desde que, assumindo que a superfície não se mova com o tempo, componentes tangentes à superfície não contribuem para mantê-la nessa configuração.
} 
Contudo, o sistema que engloba os vínculos $\phi_{0}, \phi_{1} \mathrm{e}$ $\phi_{2}$ permanece não integrável, pois além do PP não nulo entre $\phi_{1}$ e $\phi_{0}$, temos

$$
\begin{gathered}
\left\{\phi_{2}, \phi_{0}\right\}=\frac{1}{2 m}\left\{\psi, \mathbf{p}^{2}\right\}=\frac{1}{m}\{\psi, \mathbf{p}\} \cdot \mathbf{p}= \\
\frac{1}{m} \nabla \psi \cdot \mathbf{p}=\frac{2}{m} \mathbf{x} \cdot \mathbf{p},
\end{gathered}
$$

que também não é identicamente nulo. Exige-se, por conseqüência, uma nova análise de integrabilidade, que neste caso seria $d \phi_{2}=0$

$$
d \phi_{2}=\left\{\phi_{2}, \phi_{0}\right\} d t+\left\{\phi_{2}, \phi_{1}\right\} d u=\frac{2}{m} \mathbf{x} \cdot \mathbf{p} d t=0
$$

Surge, então, o vínculo

$$
\phi_{3} \equiv \frac{2}{m} \mathbf{x} \cdot \mathbf{p}=0 .
$$

O sistema permanece não integrável e ainda temos o acréscimo dos PP não nulos

$$
\begin{gathered}
\left\{\phi_{3}, \phi_{0}\right\}=\frac{1}{m^{2}}\left\{\mathbf{x} \cdot \mathbf{p}, \mathbf{p}^{2}\right\}+\frac{2}{m}\{\mathbf{x} \cdot \mathbf{p},-u \psi\}= \\
\frac{2}{m^{2}}\left(\mathbf{p}^{2}+2 m u \mathbf{x}^{2}\right), \\
\left\{\phi_{3}, \phi_{2}\right\}=\frac{2}{m}\{\mathbf{x} \cdot \mathbf{p}, \psi\}=\frac{2}{m} \mathbf{x} \cdot\{\mathbf{p}, \psi\}= \\
-\frac{2}{m} \mathbf{x} \cdot \nabla \psi=-\frac{4}{m} \mathbf{x}^{2} .
\end{gathered}
$$

Temos, então, que calcular

$$
\begin{gathered}
d \phi_{3}=\left\{\phi_{3}, \phi_{0}\right\} d t+\left\{\phi_{3}, \phi_{1}\right\} d u= \\
\frac{2}{m^{2}}\left\{\mathbf{p}^{2}+2 m u \mathbf{x}^{2}\right\} d t=0 .
\end{gathered}
$$

Assim, aparece outro vínculo

$$
\phi_{4} \equiv \frac{2}{m^{2}}\left\{\mathbf{p}^{2}+2 m u \mathbf{x}^{2}\right\}
$$

Este último também tem PP não nulos

$$
\begin{aligned}
& \left\{\phi_{4}, \phi_{0}\right\}=\frac{16 u}{m^{2}} \mathbf{x} \cdot \mathbf{p}, \\
& \left\{\phi_{4}, \phi_{1}\right\}=\frac{4}{m} \mathbf{x}^{2}\left\{u, p_{u}\right\}=\frac{4}{m} \mathbf{x}^{2}, \\
& \left\{\phi_{4}, \phi_{2}\right\}=-\frac{8}{m^{2}} \mathbf{x} \cdot \mathbf{p}, \\
& \left\{\phi_{4}, \phi_{3}\right\}=-\frac{8}{m^{3}} \mathbf{p}^{2}+\frac{16 u}{m^{2}} \mathbf{x}^{2} .
\end{aligned}
$$

Neste procedimento devemos testar a integrabilidade de $\phi_{4}$

$$
\begin{gathered}
d \phi_{4}=\left\{\phi_{4}, \phi_{0}\right\} d t+\left\{\phi_{4}, \phi_{1}\right\} d u= \\
\frac{16 u}{m^{2}} \mathbf{x} \cdot \mathbf{p} d t+\frac{4}{m} \mathbf{x}^{2} d u=0 .
\end{gathered}
$$

Contudo, algo novo aparece. Não é uma expressão do tipo $f\left(\mathbf{x}, \mathbf{p}, u, p_{u}\right)=0$, mas sim uma relação entre as diferenciais de $u$ e $t$, as variáveis independentes do sistema. As condições de integrabilidade anteriores haviam nos mostrado que a imposição de novos vínculos era necessária para que o sistema fosse integrável. A partir do quadro completo de Carathéodory essa situação indica que o conjunto original, formado pelas equações $\phi_{0}=0$ e $\phi_{1}=0$, não consistia em um conjunto completo de EDP, ou seja, a base de vetores tangentes à família de superfícies $S=\sigma$ não estava completa. As condições de integrabilidade nos mostram uma forma de completar o sistema, tomando como vínculos as expressões (59), (60), (65), (68) e (72). No entanto, ao considerarmos esses vínculos parte do formalismo devemos expandir o espaço de parâmetros para relacionar novas variáveis independentes a esses vínculos. Já temos as variáveis $t$ e $u$ referentes a $\phi_{0}$ e $\phi_{1}$, mas não existem parâmetros na teoria que possam se relacionar com os demais. Somos, portanto, obrigados a impor parâmetros adicionais na teoria, que neste caso serão chamadas $\omega, \tau$ e $\theta$. As novas variáveis independentes são, em princípio, arbitrárias. Assim, temos o conjunto de vínculos e suas respectivas variáveis independentes

$$
\begin{array}{ll}
\phi_{0}=p_{0}+H_{0} & \longrightarrow t, \\
\phi_{1}=p_{u} & \longrightarrow u, \\
\phi_{2}=\psi & \longrightarrow \omega, \\
\phi_{3}=2 / m \mathbf{p} \cdot \mathbf{x} & \longrightarrow \tau, \\
\phi_{4}=2 / m^{2}\left\{\mathbf{p}^{2}+2 m u \mathbf{x}^{2}\right\} & \longrightarrow \theta .
\end{array}
$$

No entanto, este conjunto, a princípio completo em função das condições de integrabilidade, permanece não integrável, como mostram os PP entre eles, apresentados na Tabela 1.

A novidade contida na condição sobre $\phi_{4}$ é a de que é possível encontrar uma dinâmica na qual o sistema de equações (78) seja integrável, ou seja, se as condições de integrabilidade construídas a partir da diferencial (61) não são identicamente satisfeitas, uma redefinição da diferencial pode resolver o problema. Tendo em mente essa idéia vamos considerar

$$
\begin{gathered}
d F=\left\{F, \phi_{0}\right\} d t+\left\{F, \phi_{1}\right\} d u+\left\{F, \phi_{2}\right\} d \omega+ \\
\left\{F, \phi_{3}\right\} d \tau+\left\{F, \phi_{4}\right\} d \theta
\end{gathered}
$$

As variáveis independentes $\omega, \tau$ e $\theta$ são arbitrárias a princípio. Com esta, vamos reverificar as condições de integrabilidade 
Tabela 1 - Parênteses de Poisson entre os vínculos (78)

\begin{tabular}{|c|c|c|c|c|c|c|}
\hline$\{\}$, & $\phi_{0}$ & $\phi_{1}$ & $\phi_{2}$ & & $\phi_{3}$ & $\phi_{4}$ \\
\hline$\phi_{0}$ & 0 & $-\psi$ & $-2 / m \mathbf{x} \cdot \mathbf{p}$ & $-2 / m^{2}$ & $\mathbf{p}^{2}+2 m u \mathbf{x}^{2}$ & $-16 u / m^{2} \mathbf{x} \cdot \mathbf{p}$ \\
\hline$\phi_{1}$ & $\psi$ & 0 & 0 & & 0 & $-4 / m \mathbf{x}^{2}$ \\
\hline$\phi_{0}$ & $2 / m \mathbf{x} \cdot \mathbf{p}$ & 0 & 0 & & $4 / m \mathbf{x}^{2}$ & $8 / m^{2} \mathbf{x} \cdot \mathbf{p}$ \\
\hline$\phi_{3}$ & $2 / m^{2} \mathbf{p}^{2}+2 m u \mathbf{x}^{2}$ & 0 & $-4 / m \mathbf{x}^{2}$ & & 0 & $8 / m^{3} \mathbf{p}^{2}-16 u / m^{2} \mathbf{x}^{2}$ \\
\hline & $16 u / m^{2} \mathbf{x} \cdot \mathbf{p}$ & $4 / m \mathbf{x}^{2}$ & $-8 / m^{2} \mathbf{x} \cdot \mathbf{p}$ & $-8 / m^{3} \mathbf{I}$ & $\mathbf{p}^{2}+16 u / m^{2} \mathbf{x}^{2}$ & 0 \\
\hline
\end{tabular}

$$
\begin{aligned}
d \phi_{1} & =\left\{\phi_{1}, \phi_{0}\right\} d t+\left\{\phi_{1}, \phi_{4}\right\} d \theta \\
& =\psi d t-\frac{4 \mathbf{x}^{2}}{m} d \theta=0 \\
& \therefore \quad d \theta=\frac{m \psi}{4 \mathbf{x}^{2}} d t=\frac{m}{4}\left(1-\frac{r^{2}}{\mathbf{x}^{2}}\right) d t
\end{aligned}
$$

Temos, portanto, uma equação diferencial total que nos dá a dependência de $\theta$ com relação a $t$. Com $\phi_{2}$ escrevemos

$$
\begin{aligned}
d \phi_{2} & =\left\{\phi_{2}, \phi_{0}\right\} d t+\left\{\phi_{2}, \phi_{3}\right\} d \tau+\left\{\phi_{2}, \phi_{4}\right\} d \theta \\
& =\frac{2}{m} \mathbf{x} \cdot \mathbf{p} d t+\frac{4}{m} \mathbf{x}^{2} d \tau+\frac{8}{m^{2}} \mathbf{x} \cdot \mathbf{p} d \theta=0 .
\end{aligned}
$$

Usando a relação $(80)$,

$$
\begin{gathered}
d \phi_{2}=\frac{2}{m}\left(2-\frac{r^{2}}{\mathbf{x}^{2}}\right) \mathbf{x} \cdot \mathbf{p} d t+\frac{4}{m} \mathbf{x}^{2} d \tau=0 \\
\therefore \quad d \tau=-\frac{1}{2 \mathbf{x}^{2}}\left(2-\frac{r^{2}}{\mathbf{x}^{2}}\right) \mathbf{x} \cdot \mathbf{p} d t
\end{gathered}
$$

Com o vínculo $\phi_{3}$,

Novamente usando (80),

$$
\begin{aligned}
& d \phi_{3}= \frac{2}{m^{2}}\left\{\mathbf{p}^{2}+2 m u \mathbf{x}^{2}\right\} d t+\frac{2}{m^{2}} \mathbf{p}^{2}\left(1-\frac{r^{2}}{\mathbf{x}^{2}}\right) d t- \\
& \frac{4}{m} \mathbf{x}^{2} u\left(1-\frac{r^{2}}{\mathbf{x}^{2}}\right) d t-\frac{4}{m} \mathbf{x}^{2} d \omega \\
&= \frac{2}{m^{2}}\left[\mathbf{p}^{2}\left(2-\frac{r^{2}}{\mathbf{x}^{2}}\right)+2 m u r^{2}\right] d t-\frac{4}{m} \mathbf{x}^{2} d \omega \\
& \therefore \quad d \omega=\left[\frac{1}{2 m} \frac{\mathbf{p}^{2}}{\mathbf{x}^{2}}\left(2-\frac{r^{2}}{\mathbf{x}^{2}}\right)+u \frac{r^{2}}{\mathbf{x}^{2}}\right] d t .
\end{aligned}
$$

Resta apenas o vínculo $\phi_{4}$

$$
\begin{aligned}
d \phi_{4}= & \left\{\phi_{4}, \phi_{0}\right\} d t+\left\{\phi_{4}, \phi_{1}\right\} d u+\left\{\phi_{4}, \phi_{2}\right\} d \omega+ \\
& \left\{\phi_{4}, \phi_{3}\right\} d \tau \\
= & \frac{16}{m^{2}} \mathbf{x} \cdot \mathbf{p} u d t+\frac{4}{m} \mathbf{x}^{2} d u-\frac{8}{m^{2}} \mathbf{x} \cdot \mathbf{p} d \omega \\
& -\frac{8}{m^{3}} \mathbf{p}^{2} d \tau+\frac{16}{m^{2}} \mathbf{x}^{2} u d \tau
\end{aligned}
$$

Usaremos, agora, as Eqs. (81) e (82):

$$
\begin{aligned}
& d \phi_{4}= \frac{16}{m^{2}} \mathbf{x} \cdot \mathbf{p} u d t-\frac{8}{m^{2}} \mathbf{x} \cdot \mathbf{p}\left[-\frac{1}{2 m} \frac{\mathbf{p}^{2}}{\mathbf{x}^{2}}\left(\frac{r^{2}}{\mathbf{x}^{2}}-2\right)+u \frac{r^{2}}{\mathbf{x}^{2}}\right] d t- \\
& \frac{4}{m^{3}} \frac{\mathbf{p}^{2}}{\mathbf{x}^{2}}\left(\frac{r^{2}}{\mathbf{x}^{2}}-2\right) \mathbf{x} \cdot \mathbf{p} d t+\frac{8}{m^{2}}\left(\frac{r^{2}}{\mathbf{x}^{2}}-2\right) \mathbf{x} \cdot \mathbf{p} u d t+\frac{4}{m} \mathbf{x}^{2} d u \\
& \therefore \quad d u=0 .
\end{aligned}
$$

Assim, cada condição de integrabilidade resultou em uma relação entre as variáveis independentes e o parâmetro $t$, indicando que o problema da integrabilidade não estava na completeza das equações diferenciais parciais, mas sim em assumir independência dos parâmetros. Na linguagem do quadro completo, precisamos que os vetores tangentes definidos pelos vínculos não sejam LI para que a integrabilidade se cumpra. O fato de $d u=0$ não deve ser uma surpresa nesse caso, já que $u$ é uma coordenada degenerada. A diferencial que procuramos vem a ser 


$$
d F=\left\{\left\{F, \phi_{0}\right\}+\left\{F, \phi_{2}\right\}\left[\frac{1}{2 m} \frac{\mathbf{p}^{2}}{\mathbf{x}^{2}}\left(2-\frac{r^{2}}{\mathbf{x}^{2}}\right)+u \frac{r^{2}}{\mathbf{x}^{2}}\right]-\left\{F, \phi_{3}\right\} \frac{\mathbf{x} \cdot \mathbf{p}}{2 \mathbf{x}^{2}}\left(2-\frac{r^{2}}{\mathbf{x}^{2}}\right)+\left\{F, \phi_{4}\right\} \frac{m}{4}\left(1-\frac{r^{2}}{\mathbf{x}^{2}}\right)\right\} d t
$$

O leitor pode verificar que, com essa diferencial, as condições de integrabilidade serão identicamente satisfeitas. Podemos proceder às equações de movimento, que consistem em equações para as diferenciais $d \mathbf{x}$ e $d \mathbf{p}$. Temos

$$
\begin{aligned}
d \mathbf{x} & =\left\{\mathbf{x}, \phi_{0}\right\} d t+\left\{\mathbf{x}, \phi_{1}\right\} d u+\left\{\mathbf{x}, \phi_{2}\right\} d \omega+\left\{\mathbf{x}, \phi_{3}\right\} d \tau+\left\{\mathbf{x}, \phi_{4}\right\} d \theta \\
& =\frac{1}{2 m}\left\{\mathbf{x}, \mathbf{p}^{2}\right\} d t+\frac{2}{m}\{\mathbf{x}, \mathbf{x} \cdot \mathbf{p}\} d \tau+\frac{2}{m^{2}}\left\{\mathbf{x}, \mathbf{p}^{2}\right\} d \theta=\frac{\mathbf{p}}{m} d t+\frac{2 \mathbf{x}}{m} d \tau+\frac{4 \mathbf{p}}{m^{2}} d \theta
\end{aligned}
$$

Com as Eqs. (80) e (81),

$$
d \mathbf{x}=\frac{\mathbf{x}}{m} \frac{1}{\mathbf{x}^{2}}\left(\frac{r^{2}}{\mathbf{x}^{2}}-2\right) \mathbf{x} \cdot \mathbf{p} d t+\frac{\mathbf{p}}{m}\left(2-\frac{r^{2}}{\mathbf{x}^{2}}\right) d t
$$

Nesse estágio podemos implementar os vínculos nas equações de movimento

$$
\begin{aligned}
& p_{u}=0 \\
& \psi=\mathbf{x}^{2}-r^{2}=0 \\
& \mathbf{x} \cdot \mathbf{p}=0 \\
& \mathbf{p}^{2}+2 m u \mathbf{x}^{2}=0
\end{aligned}
$$

De fato, essas equações nos dizem muito sobre o sistema. As duas primeiras são esperadas pois a equação da esfera $\mathbf{x}^{2}-r^{2}=0$ é inclusa na Lagrangiana original como um vínculo, enquanto $p_{u}$, o momento conjugado a $u$, é nulo devido à degenerescência de sua coordenada. A terceira equação nos diz que o vetor velocidade é sempre tangente à esfera. Se $\psi=0$ representa a equação de uma superfície, seu gradiente $\nabla \psi$ representa um campo vetorial ortogonal à superfície em cada ponto. A condição $\mathbf{x} \cdot \mathbf{p}=0$ também pode ser escrita por $\mathbf{v} \cdot \nabla \psi=0$, ou seja, $\mathbf{v}$ é ortogonal a $\nabla \psi$ e, por conseqüência, tangente à superfície. Note que

$$
\frac{d \psi}{d t}=2 \mathbf{x} \cdot \dot{\mathbf{x}}=\frac{2}{m} \mathbf{x} \cdot \mathbf{p}=0
$$

usando-se a terceira equação de (88). Ocorre, portanto, que a superfície, no caso a esfera, não pode se deformar com o tempo. Se a superfície se move com o tempo, essa condição já não será satisfeita e, nesse caso, a velocidade da partícula não será tangente à superfície. A última dessas condições nos fornece o parâmetro de Lagrange $u$

$$
u=-\frac{1}{2 m} \frac{\mathbf{p}^{2}}{\mathbf{x}^{2}} .
$$

Da Eq. (87), usando também as equações $\mathbf{x}^{2}=r^{2}$ $\mathrm{e} \mathbf{x} \cdot \mathbf{p}=0$, temos a equação de movimento

$$
d \mathbf{x}=\frac{\mathbf{p}}{m} d t
$$

já esperada em razão da Eq. (56). Mas a verdadeira equação dinâmica vem de

$$
\begin{aligned}
d \mathbf{p}= & \left\{\mathbf{p}, \phi_{0}\right\} d t+\left\{\mathbf{p}, \phi_{1}\right\} d u+\left\{\mathbf{p}, \phi_{2}\right\} d \omega+\left\{\mathbf{p}, \phi_{3}\right\} d \tau+ \\
& \left\{\mathbf{p}, \phi_{4}\right\} d \theta \\
= & \{\mathbf{p},-u \psi\} d t+\{\mathbf{p}, \psi\} d \omega+\frac{2}{m}\{\mathbf{p}, \mathbf{x} \cdot \mathbf{p}\} d \tau+ \\
& \frac{4}{m}\left\{\mathbf{p}, u \mathbf{x}^{2}\right\} d \theta \\
= & 2 \mathbf{x} u d t-2 \mathbf{x} d \omega-\frac{2}{m} \mathbf{p} d \tau-\frac{8}{m} \mathbf{x} u d \theta
\end{aligned}
$$

Vamos utilizar nesta última expressão as diferenciais (80), (81) e (82) em conjunto com a Eq. (88)

$$
d \mathbf{p}=-\frac{1}{m} \frac{\mathbf{p}^{2}}{\mathbf{x}^{2}} \mathbf{x} d t=-\frac{1}{m} \frac{\mathbf{p}^{2}}{r^{2}} \mathbf{x} d t
$$

A composição das Eqs. (90) e (91), derivando a primeira com relação ao tempo e usando a segunda, resulta nas equações de Euler-Lagrange para este problema

$$
\ddot{\mathbf{x}}=\frac{\dot{\mathbf{p}}}{m}=-\frac{1}{m^{2}} \frac{\mathbf{p}^{2}}{r^{2}} \mathbf{x}=-\frac{\mathbf{v}^{2}}{r^{2}} \mathbf{x} .
$$

Outro resultado importante vem a ser

$$
\frac{d}{d t}\left(\frac{1}{2} m \dot{\mathbf{x}}^{2}\right)=m \dot{\mathbf{x}} \cdot \ddot{\mathbf{x}}=-\frac{\mathbf{v}^{2}}{r^{2}} \mathbf{x} \cdot \mathbf{p}=0,
$$

com o auxílio das equações de movimento e da condição $\mathbf{x} \cdot \mathbf{p}=0$. Assim, conserva-se a energia cinética da partícula. Vale dizer que, se a superfície se mover com o tempo, essa energia não será mais uma constante de movimento e a partícula trocará energia com a própria superfície.

Segundo as equações de movimento a aceleração da partícula repousa na direção do raio da esfera, já que o vetor posição também aponta nessa direção, e o sentido é para o centro do sistema de coordenadas. A intensidade da aceleração também é conhecida, $\mathbf{v}^{2} / r$, e é a expressão que conhecemos para a aceleração centrípeta. Como o problema refere-se a uma partícula livre, não encontraremos componentes da aceleração em outras direções. As soluções dessas equações também são 
bem conhecidas: são as soluções para um oscilador harmônico em três dimensões com freqüência angular $\omega=|\mathbf{v}| / r$.

Note que a diferencial (86) resulta em $d u=d \omega=$ $d \tau=d \theta=0$, mostrando que não há dinâmica efetiva para as variáveis independentes. Neste exemplo podemos escrever

$$
d F=\left\{F, \phi_{0}\right\}^{*} d t
$$

em que

$$
\left\{F, \phi_{0}\right\}^{*}=\left\{F, \phi_{0}\right\}+\left\{F, \phi_{2}\right\}\left[\frac{1}{2 m} \frac{\mathbf{p}^{2}}{\mathbf{x}^{2}}\left(2-\frac{r^{2}}{\mathbf{x}^{2}}\right)+u \frac{r^{2}}{\mathbf{x}^{2}}\right]-\left\{F, \phi_{3}\right\} \frac{\mathbf{x} \cdot \mathbf{p}}{2 \mathbf{x}^{2}}\left(2-\frac{r^{2}}{\mathbf{x}^{2}}\right)+\left\{F, \phi_{4}\right\} \frac{m}{4}\left(1-\frac{r^{2}}{\mathbf{x}^{2}}\right) .
$$

A estrutura acima é uma particularização de uma estrutura mais geral, os Parênteses Generalizados $(\mathrm{PG})$, que definem a dinâmica no espaço de fase reduzido e podem ser definidos para todo sistema cujos vínculos resultam ser não involutivos após a exaustão das condições de integrabilidade [38]. Com isso, aprendemos que a existência de um conjunto final de vínculos não integráveis implica na redução efetiva dos graus de liberdade do sistema mediante uma refedinição da dinâmica do movimento.

\section{Um exemplo artificial}

Vamos considerar uma partícula livre em uma dimensão sujeita a um potencial que depende apenas de sua posição, cuja Lagrangiana é dada por $L=1 / 2 m v^{2}-$ $V(x)$, em que $v=\dot{x}$. Este é um sistema obviamente regular, o que pode ser facilmente verificado pela construção de sua matriz Hessiana. Contudo, vamos redefinir esta variável tal que $x \longrightarrow x_{1}+x_{2}$ e a Lagrangiana tome a forma [24]

$$
L\left(x_{1}, x_{2}, v_{1}, v_{2}\right)=\frac{1}{2} m\left(v_{1}+v_{2}\right)^{2}-V\left(x_{1}+x_{2}\right) .
$$

Note que o sistema permanece inalterado, mas ao usarmos a Lagrangiana (95) estamos inserindo no sistema um grau de liberdade desnecessário. É este grau de liberdade a mais que introduzirá o que chamaremos "liberdade de gauge" ao sistema.

Os momentos conjugados são dados por

$$
p_{1}=\frac{\partial L}{\partial v_{1}}=m\left(v_{1}+v_{2}\right) \quad p_{2}=\frac{\partial L}{\partial v_{2}}=m\left(v_{1}+v_{2}\right)
$$

que nos fornecem a matrix Hessiana

$$
W \equiv m\left(\begin{array}{ll}
1 & 1 \\
1 & 1
\end{array}\right)
$$

singular de posto 1 .

Ao tentarmos escrever as velocidades em função dos momentos encontramos o vínculo

$$
p_{1}-p_{2}=0 \text {. }
$$

Vamos calcular a Hamiltoniana canônica em função de $p_{1}$

$$
H_{0} \equiv p_{1} v_{1}+p_{2} v_{2}-L=\frac{\left(p_{1}\right)^{2}}{2 m}+V\left(x_{1}+x_{2}\right) .
$$

Assim, temos o sistema de HJ formado pelos vínculos

$$
\begin{aligned}
\phi_{0} & \equiv p_{0}+H_{0}=0 \\
\phi_{1} & \equiv p_{1}-p_{2}=0
\end{aligned}
$$

O parâmetro de evolução que corresponde ao vínculo $\phi_{0}$ é, como já sabemos, o tempo. Já o parâmetro de evolução que corresponde ao vínculo $\phi_{1}$ pode ser escolhido de acordo com as variáveis da Hamiltoniana. Como decidimos escrever a Hamiltoniana em função de $p_{1}$ o parâmetro deve ser $x_{2}$. Assim, a evolução do sistema é dada de acordo com a diferencial

$$
d F=\left\{F, \phi_{0}\right\} d t+\left\{F, \phi_{1}\right\} d x_{2} .
$$

Note que

$$
\left\{\phi_{0}, \phi_{1}\right\}=\left\{H_{0}, p_{1}-p_{2}\right\}=\frac{\partial V}{\partial x_{1}}-\frac{\partial V}{\partial x_{2}}=0,
$$

visto que $V$ depende apenas da soma $x_{1}+x_{2}$. Assim, o sistema de HJ é um conjunto de vínculos já em involução, o que, como conseqüência, implica na satisfação das condições de integrabilidade $d \phi_{0}=0 \mathrm{e}$ $d \phi_{1}=0$.

Se o sistema é integrável tal como se apresenta, não há mais nada a fazer a não ser calcular as equações de movimento a partir de (100)

$$
\begin{aligned}
d x_{1}= & \left\{x_{1}, \phi_{0}\right\} d t+\left\{x_{1}, \phi_{1}\right\} d x_{2}=\frac{p_{1}}{m} d t+d x_{2} \\
& v_{1}=\frac{p_{1}}{m}+v_{2} \\
d p_{1}= & \left\{p_{1}, \phi_{0}\right\} d t+\left\{p_{1}, \phi_{1}\right\} d x_{2}=-\frac{\partial V}{\partial x_{1}} d t
\end{aligned}
$$

em que a velocidade $v_{2}$ é arbitrária.

Note que a equação de movimento (102) é uma equação canônica puramente cinemática. A equação que define a dinâmica do sistema é dada pela Eq. (103), 
que não depende da velocidade $v_{2}$. A interpretação deste fato é que o grau de liberdade extra, representado pela variável $x_{2}$ por nossa escolha, não interfere na dinâmica do sistema.

Teorias de gauge, como o eletromagnetismo, apresentam um ou mais do mesmo tipo de vínculo dado por $\phi_{1}$ acima, ou seja, vínculos que se encontram em involução, satisfazendo identicamente suas condições de integrabilidade. Nesses sistemas as velocidades relativas às variáveis independentes são arbitrárias. Contudo, a dinâmica relativa a essas variáveis, chamadas variáveis de gauge, não interferem na evolução temporal do sistema.

\section{O campo eletromagnético livre}

Vamos para um caso real em que aparecem vínculos em involução. O campo eletromagnético livre é o exemplo mais simples de liberdade de gauge encontrado na natureza. Sistemas em teoria de campos são diferentes dos sistemas em mecânica clássica essencialmente por possuírem infinitos graus de liberdade: as coordenadas do espaço de configuração são campos que são funções de parâmetros contínuos, no geral pontos do espaço-tempo. No caso do campo eletromagnético existem quatro campos $A_{0}(x)$ e $A_{i}(x)$, o primeiro chamado campo escalar e os demais componentes do potencial vetor. Denominaremos por letras latinas $x$ um ponto $\left(x^{0}, x^{1}, x^{2}, x^{3}\right)$ do espaço-tempo. A métrica é a de Minkowski $\eta_{\alpha \beta}=\operatorname{diag}\left(\eta_{00} \eta_{11} \eta_{22} \eta_{33}\right)$.

Diferentemente da mecânica clássica, a mecânica de campos é independente do parâmetro, ou seja, as curvas que representam a dinâmica no espaço de configuração são funções de um parâmetro arbitrário. No geral, o tempo próprio, ou mesmo o comprimento de arco, é usado para parametrizar as soluções. Contudo, para que o formalismo que desenvolvemos até aqui não precise ser modificado para permitir escolhas livres, vamos fazer opção pela coordenada ${ }^{13} x^{0}=c t$ como parâmetro de evolução.

A ação do campo EM sem fontes vem a ser o funcional

$$
\begin{aligned}
& S=-\frac{1}{4} \int F_{\alpha \beta}(x) F^{\alpha \beta}(x) d^{4} x, \\
& F_{\alpha \beta}(x)=\partial_{\alpha}^{x} A_{\beta}(x)-\partial_{\beta}^{x} A_{\alpha}(x) .
\end{aligned}
$$

Em nossa notação $\partial_{\alpha}^{x} \equiv \partial / \partial x^{\alpha}$ é a derivada parcial aplicada no ponto $x$. Temos, assim, a função Lagrangiana

$$
L=-\frac{1}{4} \int F_{\alpha \beta}(x) F^{\alpha \beta}(x) d^{3} x .
$$

Podemos escrever

$$
L=\int \mathcal{L}(x) d^{3} x,
$$

em que identificamos a densidade Lagrangiana

$$
\mathcal{L}(x)=-\frac{1}{4} F_{\alpha \beta}(x) F^{\alpha \beta}(x) .
$$

Vamos encontrar os momentos conjugados às variáveis $A_{\alpha}$. Como o parâmetro escolhido é a coordenada $x^{0}$, as velocidades relativas às variáveis são dadas por $\dot{A_{\alpha}}=\partial_{0} A_{\alpha}$. Assim, temos as equações

$$
\begin{aligned}
\pi^{0}(x) & =\int \frac{\delta \mathcal{L}(y)}{\delta \partial_{0} A_{0}(x)} d^{3} y=0 \\
\pi^{i}(x) & =\int \frac{\delta \mathcal{L}(y)}{\delta \partial_{0} A_{i}(x)} d^{3} y=-\frac{1}{4} \int 2\left[\frac{\delta F_{\alpha \beta}(y)}{\delta \partial_{0} A_{i}(x)} F^{\alpha \beta}(y)\right] d^{3} y=-\frac{1}{2} \int\left(\delta_{\alpha}^{0} \delta_{\beta}^{i}-\delta_{\beta}^{0} \delta_{\alpha}^{i}\right) F^{\alpha \beta}(y) \delta^{3}(x-y) d^{3} y \\
& =\int F^{i 0}(y) \delta^{3}(x-y) d^{3} y=F^{i 0}(x)
\end{aligned}
$$

Usaremos o símbolo $\delta$ para derivadas funcionais, além de representar as deltas de Kroenecker e Dirac. Derivando novamente esses momentos com relação às velocidades podemos construir a matriz Hessiana

$$
W^{i j}=-\eta^{00} \eta^{i k}\left(\begin{array}{cc}
0_{1 \times 1} & 0_{1 \times 3} \\
0_{3 \times 1} & \delta_{k}^{j}
\end{array}\right) \delta^{3}(x-y) .
$$

Essa matriz é singular e possui três autovalores diferentes de zero, ou seja, tem posto 3 . Uma das velo- cidades não pode ser invertida. É o caso da velocidade $\dot{A}_{0}$ e, por essa razão, temos o vínculo

$$
\phi^{0}(x) \equiv \pi^{0}(x)=0 .
$$

Por outro lado as velocidades $\dot{A}_{i}$ podem ser escritas em função das variáveis e dos momentos ${ }^{14}$ :

$$
\partial_{0} A_{i}=\partial_{i} A_{0}-\eta_{00} \eta_{i j} \pi^{j}
$$

A singularidade da Hessiana indica a separação entre as variáveis dependentes $\left(A_{i}\right)$ e as independen-

\footnotetext{
${ }^{13}$ Usaremos $c=1$ neste exemplo.

${ }^{14} \mathrm{~A}$ partir deste ponto passaremos a omitir o ponto de aplicação das funções no espaço-tempo sempre que este for trivial.
} 
tes $\left(A_{0}, t\right)$. Além da Eq. (109) o sistema obedece à equação

$$
\phi^{t} \equiv p^{t}+H\left(A_{\alpha}, \pi^{i}\right)=0, \quad p^{t}=\partial_{0} S,
$$

com a qual temos a função Hamiltoniana canônica

$$
\begin{gathered}
H=\int\left(\dot{A}_{0} \pi^{0}+\dot{A}_{i} \pi^{i}+\frac{1}{4} F_{\alpha \beta} F^{\alpha \beta}\right) d^{3} x= \\
\int\left(-\frac{1}{2} \eta_{00} \eta_{i j} \pi^{i} \pi^{j}+\pi^{i} \partial_{i} A_{0}+\frac{1}{4} F_{i j} F^{i j}\right) d^{3} x .
\end{gathered}
$$

Podemos escrever a densidade Hamiltoniana canônica

$$
\begin{gathered}
\mathcal{H}(x)=-\frac{1}{2} \eta_{00} \eta_{i j} \pi^{i} \pi^{j}+\pi^{i} \partial_{i} A_{0}+\frac{1}{4} F_{i j} F^{i j}, \\
F_{i j}(x)=\partial_{i}^{x} A_{j}(x)-\partial_{j}^{x} A_{i}(x) .
\end{gathered}
$$

Existem, portanto, duas equações

$$
\begin{gathered}
\phi^{0}(x)=\pi^{0}(x)=0, \\
\phi^{t}(x)=p^{t}(x)+\mathcal{H}(x)=0,
\end{gathered}
$$

uma para cada variável independente.

Precisamos impor condições de integrabilidade a esses vínculos pois eles claramente não estão em involução. Para que o formalismo de teoria de campos tenha sentido devemos definir os PP pela estrutura

$$
\{F(x), G(y)\}=\int d^{3} z\left[\frac{\delta F(x)}{\delta A_{\mu}(z)} \frac{\delta G(y)}{\delta \pi^{\mu}(z)}-\frac{\delta G(y)}{\delta A_{\mu}(z)} \frac{\delta F(x)}{\delta \pi^{\mu}(z)}\right]_{x^{0}=\text { constante }} .
$$

A dinâmica é dada pela diferencial

$$
d F=\int d^{3} x\left\{F, \phi^{t}(x)\right\} d t+\int d^{3} x\left\{F, \phi^{0}(x)\right\} d A_{0}(x)
$$

Vamos começar por $\phi^{0}$

$$
\begin{aligned}
d \phi^{0}(x) & =\int d^{3} y\left\{\phi^{0}(x), \phi^{t}(y)\right\} d t+\int d^{3} y\left\{\phi^{0}(x), \phi^{0}(y)\right\} d A_{0}(y) \\
& =\int d^{3} y\left\{\pi^{0}(x), \mathcal{H}(y)\right\} d t=-\int d^{3} y \frac{\delta \mathcal{H}(y)}{\delta A_{0}(x)} d t \\
& =-\int d^{3} y \pi^{i}(y) \partial_{i}^{y} \delta^{3}(x-y) d t=\int d^{3} y \partial_{i}^{y} \pi^{i}(y) \delta^{3}(x-y) d t=\partial_{i}^{x} \pi^{i}(x) d t=0
\end{aligned}
$$

que resulta na expressão $\partial_{i} \pi^{i}=0$, formulada como um novo vínculo

$$
\phi^{1} \equiv \partial_{i} \pi^{i}=0
$$

A condição $d \phi^{1}=0$ resulta em

$$
\begin{aligned}
d \phi^{1} & =\int d^{3} y\left\{\phi^{1}(x), \phi^{t}(y)\right\} d t+\int d^{3} y\left\{\phi^{1}(x), \phi^{0}(y)\right\} d A_{0}(y) \\
& =\int d^{3} y\left\{\partial_{i} \pi^{i}(x), \mathcal{H}(y)\right\} d t=\int d^{3} y \partial_{i}^{x}\left[\frac{\delta \mathcal{H}(y)}{\delta A_{i}(x)}\right] d t \\
& =\int d^{3} y \partial_{i}^{x}\left[F^{j i}(y) \partial_{j}^{y} \delta^{3}(x-y)\right] d t=\int d^{3} y F^{j i}(y) \partial_{i}^{x} \partial_{j}^{y} \delta^{3}(x-y) d t \\
& =\int d^{3} y \partial_{i}^{x} \partial_{j}^{y} F^{j i}(y) \delta^{3}(x-y) d t=\partial_{i} \partial_{j} F^{j i} d t
\end{aligned}
$$

A expressão à direita é identicamente nula pois $F^{i j}$ é antissimétrico ${ }^{15}$. Nesse caso não há outros vínculos a descobrir. Ficamos, por fim, com o conjunto

$$
\begin{aligned}
& \phi^{t}(x)=p^{t}(x)+\mathcal{H}(x), \\
& \phi^{0}(x)=\pi^{0}(x), \\
& \phi^{1}(x)=\partial_{i} \pi^{i}(x) .
\end{aligned}
$$

\footnotetext{
${ }^{15} \partial_{i} \partial_{j} F^{j i}=1 / 2 \partial_{i} \partial_{j}\left[F^{j i}-F^{i j}\right]=1 / 2 \partial_{i} \partial_{j} F^{j i}-1 / 2 \partial_{i} \partial_{j} F^{i j}=1 / 2 \partial_{i} \partial_{j} F^{j i}-1 / 2 \partial_{j} \partial_{i} F^{i j}=1 / 2 \partial_{i} \partial_{j} F^{j i}-1 / 2 \partial_{i} \partial_{j} F^{j i}=0$.
} 
Não precisamos impor a condição sobre $\phi^{t}$ pois podemos ver claramente que o conjunto acima está em involução. Da mesma forma que no exemplo da partícula livre na esfera, devemos adicionar uma nova variável independente, desta vez arbitrária, ao conjunto $\left(t, A_{0}\right)$. Relacionaremos a variável $\omega$ ao vínculo $\phi^{1}$. Nesse caso a diferencial de uma função dinâmica é dada por

$$
d F=\int d^{3} x\left\{F, \phi^{t}(x)\right\} d t+\int d^{3} x\left\{F, \phi^{0}(x)\right\} d A_{0}(x)+\int d^{3} x\left\{F, \phi^{1}(x)\right\} d \omega .
$$

Assim, temos como variáveis dependentes os campos $A_{i}$ e como variáveis independentes $t, A_{0}$ e $\omega$, este último um parâmetro arbitrário. As equações de movimento são dadas por

$$
\begin{aligned}
d A_{i}(x) & =\int d^{3} y\left\{A_{i}(x), \phi^{t}(y)\right\} d t+\int d^{3} y\left\{A_{i}(x), \phi^{0}(y)\right\} d A_{0}(y)+\int d^{3} y\left\{A_{i}(x), \phi^{1}(y)\right\} d \omega \\
& =\int d^{3} y \frac{\delta \mathcal{H}(y)}{\delta \pi^{i}(x)} d t+\int d^{3} y \frac{\delta \partial_{j}^{y} \pi^{j}(y)}{\delta \pi^{i}(x)} d \omega=\left[\partial_{i} A_{0}-\eta_{00} \eta_{i j} \pi^{j}\right] d t+\int \partial_{i}^{y} \delta^{3}(x-y) d^{3} y d \omega
\end{aligned}
$$

Da teoria de distribuições [23]

$$
\int \partial_{i}^{y} \delta^{3}(x-y) d^{3} y=-\int \partial_{i}^{x} \delta^{3}(x-y) d^{3} y=-\partial_{i}^{x} \int \delta^{3}(x-y) d^{3} y=0 .
$$

Temos, com este resultado,

$$
\partial_{0} A_{i}=\partial_{i} A_{0}-\eta_{00} \eta_{i j} \pi^{j} \quad \longrightarrow \quad \pi^{i}=F^{i 0}
$$

resultado que já conhecemos.

O segundo par de equações características vem a ser

$$
\begin{aligned}
d \pi^{i} & =\int d^{3} y\left\{\pi^{i}(x), \phi^{t}(y)\right\} d t+\int d^{3} y\left\{\pi^{i}(x), \phi^{0}(y)\right\} d A_{0}(y)+\int d^{3} y\left\{\pi^{i}(x), \phi^{1}(y)\right\} d \omega \\
& =-\int d^{3} y \frac{\delta \mathcal{H}(y)}{\delta A_{i}(x)} d t=-\frac{1}{2} \int d^{3} y \frac{\delta F_{j k}(y)}{\delta A_{i}(x)} F^{j k}(y) d t=-\frac{1}{2} \int d^{3} y\left[\delta_{k}^{i} \partial_{j}^{y} \delta^{3}(x-y)-\delta_{j}^{i} \partial_{k}^{y} \delta^{3}(x-y)\right] F^{j k}(y) d t \\
& =\frac{1}{2} \int d^{3} y\left[\partial_{j}^{y} F^{j i}(y)-\partial_{k}^{y} F^{i k}(y)\right] \delta^{3}(x-y) d t=\partial_{j} F^{j i} d t
\end{aligned}
$$

Essa última expressão podemos escrever por

$$
\partial_{0} \pi^{i}+\partial_{j} F^{i j}=0 .
$$

Se usarmos a Eq. (123) temos

$$
\partial_{0} F^{i 0}+\partial_{j} F^{i j}=0 \quad \longrightarrow \quad \partial_{\alpha} F^{i \alpha}=0 .
$$

Assim, temos as equações de movimento (126) em conjunto com as Eqs. (123). Se usarmos a expressão $\partial_{i} \pi^{i}=0$

$$
\partial_{\alpha} F^{0 \alpha}=0
$$

Note que podemos unificar as Eqs. (126) e (127)

$$
\partial_{\alpha} F^{\beta \alpha}=0 \text {. }
$$

Essas equações formam o primeiro par de equações de Maxwell sem fontes.

O campo eletromagnético é o mais simples exemplo de teoria com liberdade de gauge, caracterizado pela presença de um conjunto final de vínculos em involução.
Note que o procedimento, diferentemente do caso da partícula livre restrita a uma superfície, não foi capaz de reduzir os graus de liberdade da teoria. Em vez disso, a dinâmica dos graus de liberdade referentes às variáveis $A_{0}$ e $\omega$ permanece arbitrária.

\section{Considerações finais}

Nas duas partes deste trabalho fomos capazes de analisar o formalismo de Hamilton-Jacobi sob o geometricamente rico escopo de Carathéodory. Vimos que a existência de uma equação diferencial parcial é natural nos sistemas dinâmicos que obedecem ao princípio de ação estacionária e que as soluções, que são famílias de superfícies no espaço de configuração do sistema, estão relacionadas às trajetórias dinâmicas no espaço de fase a partir das equações características. No caso de sistemas regulares as equações características são as já conhecidas equações de Hamilton. Já no caso de sistemas singulares, a análise de equações de movimento passa pela definição dos vínculos da teoria, já que a sin- 
gularidade da matriz Hessinana vem da existência de relações entre coordenadas e momentos que restringem, a princípio, os graus de liberdade do sistema.

Nessa segunda parte nos preocupamos em analisar como o formalismo de HJ é capaz de lidar de forma natural com a existência de vínculos em sistemas Lagrangianos. Neste contexto, a análise de sistemas singulares é relativamente nova: os primeiros trabalhos estendendo a formulação desenvolvida por Carathéodory para o tratamento de sistemas singulares foram feitos por Güler em 1992 [25, 26]. Outras extensões foram realizadas por outros autores e, hoje, é bem estabelecido como são descritos em HJ os sistemas singulares com derivadas de ordem superior [34-36], sistemas singulares descritos por variáveis de Berezin [37], sistemas descritos por ações de primeira ordem [38], entre outros. Desde o desenvolvimento inicial feito por Güler, diversas aplicações a sistemas singulares têm sido realizadas, [27-33]. Vamos resumir o que vimos nesse estudo.

Vínculos em sistemas singulares surgem da definição dos momentos conjugados às coordenadas. A singularidade da matriz Hessiana implica na impossibilidade de se escrever certas velocidades, senão todas, em função das coordenadas e dos momentos, comprometendo, a princípio, a definição de uma equação de HJ para o sistema. Contudo, uma equação de HJ pode ser definida univocamente para uma região do espaço de configuração em que os vínculos sejam impostos como condições sobre o sistema. Além disso, os próprios vínculos se tornam equações diferenciais parciais. Um conjunto de equações, que abrange os vínculos e a equação de HJ, pode ser definido como o conjunto de EPD de HJ e é sobre este conjunto que as equações características são construídas. As características definem, por si, um conjunto de variáveis dependentes relacionados ao espaço de fase reduzido $\left(q^{a}, p_{a}\right)$, onde a dinâmica do sistema ocorre de fato, e um conjunto de variáveis independentes $q^{\alpha} \equiv\left(t, q^{z}\right)$, que são os parâmetros da teoria. No espaço de fase reduzido podemos definir parênteses de Poisson que geram univocamente a dinâmica do sistema.

Contudo, as considerações acima são válidas considerando um conjunto completo de EDP, cujas características são completamente integráveis, condição que, em geral, não é satisfeita em princípio nos sistemas físicos. Condições de integrabilidade devem ser testadas e elas exigem que os vínculos estejam em involução com os PP para que o conjunto seja completo. Do teste das condições de integrabilidade $d \phi_{\alpha}=0$ três situações podem ocorrer. Uma delas é que o conjunto de vínculos, ou um subconjunto deles, esteja realmente em involução, resultando em relações do tipo $0=0$ ou em vínculos já existentes. Neste caso o conjunto em involução é completo. Pode ocorrer também que um conjunto, ou a totalidade dos vínculos, não se encontrem em involução. Como resultado as condições de integrabilidade tornam-se relações algébricas entre as di- ferenciais das variáveis independentes. Se este conjunto de equações algébricas tiver solução única, podemos redefinir a dinâmica deste sistema. A terceira possibilidade é o caso em que as condições de integrabilidade resultem em relações entre as variáveis dinâmicas. Essas relações devem ser impostas na teoria como novos vínculos, adicionadas ao conjunto original e condições de integrabilidade sobre esses vínculos devem também ser impostas.

Os exemplos que trabalhamos servem para clarificar como proceder com o formalismo a partir da análise das condições de integrabilidade. A partícula livre na esfera nos fornece um exemplo em que as condições impõem novos vínculos na teoria. Depois que todos os vínculos são encontrados é necessário considerá-los como condições sobre o sistema, o que é feito introduzindo novas variáveis independentes e uma nova diferencial fundamental (79). No caso deste exemplo os vínculos não formam um sistema em involução e as condições de integrabilidade nos mostram que uma redefinição da dinâmica, de acordo com a diferencial (86), torna o sistema completamente integrável. Um grande resultado desse formalismo é o fato de que essa redefinição na dinâmica implica na definição de parênteses generalizados [38], que possuem as mesmas propriedades dos PP e que definem a dinâmica no espaço de fase reduzido.

No exemplo da seção 7 introduzimos artificialmente um grau de liberdade desnecessário ao sistema de partícula unidimensional com potencial dependente da posição. Este é o mais simples exemplo concebível de sistema mecânico com liberdade de gauge. Nessa teoria temos dois vínculos, $\phi_{0}$ e $\phi_{1}$, que estão automaticamente em involução e, portanto, já formam um sistema completo com equações características integráveis. Neste caso a análise não é capaz de eliminar a variável independente $x_{2}$ do sistema, o que seria esperado se o conjunto resultasse ser não involutivo em razão do aparecimento de uma expressão do tipo $d x_{2}=g(x, p) d t$. Contudo, a dinâmica na direção desta variável não é física: qualquer evolução na direção desse grau de liberdade não muda a evolução temporal do sistema, o que é característica de teorias de gauge.

O exemplo mais simples de teoria de gauge que sabemos existir na natureza é o campo eletromagnético. Como vimos, a condição de integrabilidade sobre o vínculo $\pi^{0}=0$ resulta na imposição da lei de Gauss $\partial_{i} \pi^{i}=\partial_{i} F^{i 0}=\nabla \cdot \mathbf{E}=0$ como novo vínculo. O conjunto completo é involutivo e, portanto, tem equações características completamente integráveis. A evolução de um observável físico é dada pela diferencial (122), que não resolve os graus de liberdade físicos, a exemplo do caso anterior. Contudo, a evolução na direção das variáveis independentes $A_{0}$ e $\omega$ não pode ser considerada física. Essas duas variáveis são chamadas variáveis de gauge.

Do campo eletromagnético, sabemos que apesar de 
ser descrito por quatro variáveis, $A_{0}$ e $A_{i}$, apenas dois graus de liberdade são físicos. Para resolver esses graus de liberdade é necessária uma fixação de gauge, o que foge ao escopo deste trabalho. Mas sabemos desde o início que $A_{0}$ não é um grau de liberdade físico, já que é uma variável ignorável na Lagrangiana. Já $\omega$, contudo, é um parâmetro arbitrário e não saberemos qual é o seu significado físico até que um gauge seja escolhido para resolver os graus de liberdade. Podemos deduzir que, dependendo da escolha, $\omega$ pode assumir a identidade de uma das variáveis restantes ou mesmo de uma combinação linear delas.

O passo natural para o estudo do formalismo de HJ em sistemas vinculados a partir desse ponto é estudar as relações entre sistemas com vínculos em involução e simetrias, tanto no aspecto clássico quanto no aspecto quântico dessas teorias.

\section{Agradecimentos}

M.C. Bertin agradece à CAPES pelo suporte integral. B.M. Pimentel agradece ao CNPq pelo suporte parcial. P.J. Pompeia agradece ao CNPq pelo suporte e ao CTA pelo apoio.

\section{Referências}

[1] M.C. Bertin, B M. Pimentel and P.J. Pompeia, Revista Brasileira de Ensino de Física 29, 393 (2007).

[2] P.A.M. Dirac, Canad. J. Math. 2, 129 (1950).

[3] J.L. Anderson and P.G. Bergmann, Phys. Rev. 111, 965 (1951).

[4] P.A.M. Dirac, Canad. J. Math. 3, 1 (1951).

[5] P.G. Bergmann and I. Goldberg, Phys. Rev. 98, 531 (1955).

[6] P.A.M. Dirac, Proc. Roy. Soc. A246, 326 (1958).

[7] P.A.M. Dirac, Lectures in Quantum Mechanics (Yeshiva University, New York, 1964).

[8] W. Kundt, Springer Tracts in Modern Physics 40, 107 (1966).

[9] S. Shanmugadhasan, Proc. Camb. Philos. Soc. 59, 743 (1963).

[10] S. Shanmugadhasan, J. Math. Phys. 14, 667 (1973).

[11] A. Hanson, T. Regge and C. Teitelboim, Constrained Hamiltonian Systems (Acc. Naz. dei Licei, Roma, 1976).

[12] K. Sundermeyer, Constrained Dynamics, Lecture Notes in Physics n. 169 (Springer, Nova York, 1982).

[13] D.M. Gitman and I.V. Tyutin, Quantization of Fields with Constraints (Springer-Verlag, New York/Berlin, 1990).
[14] K.R. Symon, Mecânica (Editora Campos Ltda, Rio de Janeiro, 1982).

[15] H. Goldstein, Classical Mechanics (Addison-Wesley, Cambridge, 1950).

[16] C. Lanczos, The Variational Principles of Mechanics (Dover, Nova York, 1986), $4^{\text {th }}$ ed.

[17] J.V. José and E.J. Saletan, Classical Dynamics, A Contemporary Approach (Cambridge Univ. Press, Cambridge, 1998).

[18] D.M. Gitman and I.V. Tyutin, Nucl. Phys. B, 630509 (2002).

[19] R. Courant and D. Hilbert, Methods of Mathematical Physics, v. II (John Wiley and Sons, Nova York, 1962).

[20] C. Carathéodory, Calculus of Variations and Partial Differential Equations of the First Order - Part I, II (Holden Day, Inc, San Francisco, 1967).

[21] T. Frankel, The Geometry of Physics, an Introduction (Cambridge Univ. Press, Cambridge, 1997).

[22] B. Schutz, Geometrical Methods of Mathematical Physics (Cambridge Univ. Press, Cambridge, 1980).

[23] C.L.R. Braga, Notas de Física Matemática - Equações Diferenciais, Funções de Green e Distribuições (Editora Livraria da Física USP, São Paulo, 2006).

[24] H. Matschull, Dirac's Canonical Quantization Programme, quant-ph/9606031 (1996).

[25] Y. Güler, Il Nuovo Cimento B 107, 1398 (1992).

[26] Y. Güler, Il Nuovo Cimento B 107, 1143 (1992).

[27] Y. Güler, Il Nuovo Cimento B 109, 341 (1994).

[28] Y. Güler, Il Nuovo Cimento B 111, 513 (1996).

[29] Y. Güler and D. Baleanu, Il Nuovo Cimento B 114, 1023 (1999).

[30] Y. Güler and D. Baleanu, Il Nuovo Cimento B 115, 319 (2000).

[31] Soon-Tae Hong, Yong-Wan Kim, Young-Jai Park and K.D. Rothe, Mod. Phys. Lett. A 17, 435 (2002).

[32] B.M. Pimentel, P.J. Pompeia, J.F. da Rocha-Neto and R.G. Teixeira, Gen. Rel. Grav. 35, 877 (2003).

[33] B.M. Pimentel, P.J. Pompeia and J.F. da Rocha-Neto, Il Nuovo Cimento B 120, 981 (2005).

[34] B.M. Pimentel and R.G. Teixeira, Il Nuovo Cimento B 111, 841 (1996).

[35] B.M. Pimentel and R.G. Teixeira, Il Nuovo Cimento B 113, 805 (1998).

[36] M.C. Bertin, B.M. Pimentel and P.J. Pompeia, Ann. Phys. 323, 527 (2008).

[37] B.M. Pimentel, R.G. Teixeira and J.L. Tomazelli Ann. Phys. 267, 75 (1998).

[38] M.C. Bertin, B.M. Pimentel and P.J. Pompeia, Mod. Phys. Lett. A 20, 2873 (2005). 